\title{
Laparo-endoscopic versus open recurrent inguinal hernia repair: should we follow the guidelines?
}

\author{
F. Köckerling ${ }^{1} \cdot$ R. Bittner ${ }^{2} \cdot$ A. Kuthe ${ }^{3} \cdot$ B. Stechemesser ${ }^{4} \cdot$ R. Lorenz ${ }^{5} \cdot$ \\ A. Koch ${ }^{6}$ W. Reinpold ${ }^{7}$ H. Niebuhr ${ }^{8} \cdot$ M. Hukauf ${ }^{9} \cdot$ C. Schug-Pass ${ }^{1}$
}

Received: 19 August 2016/ Accepted: 9 November 2016/Published online: 8 December 2016

(C) The Author(s) 2016. This article is published with open access at Springerlink.com

\begin{abstract}
Introduction On the basis of six meta-analyses, the guidelines of the European Hernia Society (EHS) recommend laparo-endoscopic recurrent repair following previous open inguinal hernia operation and, likewise, open repair following previous laparo-endoscopic operation. So far no data are available on implementation of the guidelines or for comparison of outcomes. Besides, there are no studies for comparison of outcomes for compliance versus non-compliance with the guidelines.
\end{abstract}

F. Köckerling

ferdinand.koeckerling@vivantes.de

1 Department of Surgery and Center for Minimally Invasive Surgery, Academic Teaching Hospital of Charité Medical School, Vivantes Hospital, Neue Bergstrasse 6, 13585 Berlin, Germany

2 Hernia Center, Winghofer Medicum, Winghofer Strasse 42, 72108 Rottenburg am Neckar, Germany

3 Department of General and Visceral Surgery, German Red Cross Hospital, Lützerodestrasse 1, 30161 Hannover, Germany

4 Hernia Center Cologne, PAN - Hospital, Zeppelinstrasse 1, 50667 Cologne, Germany

5 3Surgeons, Klosterstrasse 34/35, 13581 Berlin, Germany

6 Hernia Center Cottbus, Gerhard-Hauptmann-Strasse 15, 03044 Cottbus, Germany

7 Department of Surgery and Hernia Center, Wilhelmsburg Hospital Gross-Sand, Gross-Sand 3, 21107 Hamburg, Germany

8 Hanse-Hernia Center, Alte Holstenstrasse 16, 21031 Hamburg, Germany

9 StatConsult GmbH, Halberstädter Strasse 40 a, 39112 Magdeburg, Germany
Patients and methods In total, 4812 patients with elective unilateral recurrent inguinal hernia repair in men were enrolled between September 1, 2009, and September 17, 2014, in the Herniamed Registry. Only patients with 1-year follow-up were included.

Results Out of the 2482 laparo-endoscopic recurrent repair operations $90.5 \%$ of patients, and out of the 2330 open recurrent repair procedures only $38.5 \%$ of patients, were operated on in accordance with the guidelines of the EHS. Besides, on compliance with the guidelines multivariable analysis demonstrated for laparo-endoscopic recurrent repair a significantly lower risk of pain at rest (OR 0.643 [0.476; 0.868]; $p=0.004$ ) and pain on exertion (OR 0.679 [0.537; 0.857]; $p=0.001)$. Comparison of laparo-endoscopic and open recurrent repair in settings of compliance versus non-compliance with the guidelines showed a higher incidence of perioperative complications and re-recurrences for recurrent repairs that did not comply with the guidelines.

Conclusion The EHS guidelines for recurrent inguinal hernia repair are not yet being observed to the extent required. Non-compliance with the guidelines is associated with higher perioperative complication rates and higher risk of re-recurrence. Even on compliance with the guidelines, the risk of pain at rest and pain on exertion is higher after open recurrent repair than after laparo-endoscopic repair.

Keywords Inguinal hernia - Recurrence Postoperative complications $\cdot$ Pain $\cdot$ Endoscopic repair

Compared with primary inguinal hernia operations, both open and laparo-endoscopic recurrent repair procedures are associated with a higher rate of perioperative 
complications, re-recurrences and chronic pain [1, 2]. Six meta-analyses are available for comparison of laparo-endoscopic with open recurrent inguinal hernia repairs [3-8]. These meta-analyses analyzed 12 studies [9-20]. Compared with the meta-analysis by $\mathrm{Li}$ et al. [7], which included non-randomized studies [12, 13, 16, 19], the meta-analysis by Pisanu et al. [6] featured the largest number of exclusively prospective randomized studies $[9,11,14,15,17,18,20]$. There was no high risk of bias in any of the included trials [6]. The studies included in total 647 patients with recurrent inguinal hernia randomized to either laparo-endoscopic repair $[n=333 ; 51.5 \%$, transabdominal preperitoneal patch plasty (TAPP) and totally extraperitoneal patch plasty (TEP)], or anterior open repair ( $n=314 ; 48.5 \%$, by Lichtenstein technique). Patients who underwent laparo-endoscopic repair experienced significantly less chronic pain (9.2 vs $21.5 \%$; $p=0.003$ ). Patients of the laparo-endoscopic group had a significantly earlier return to normal daily activities (13.9 vs 18.4 days, SMD $-0.68,95 \%$ CI -0.94 to $-0.43 ; p<0.000001)$. Operative time was significantly longer in laparo-endoscopic operations (62.9 vs $54.2 \mathrm{~min}$, SMD 0.46, 95\% CI 0.03, 0.89; $p=0.04$ ) [6]. No other differences were found [6]. Another prospective randomized controlled study that was not included in the meta-analyses also identified a lower chronic pain rate after laparo-endoscopic recurrent repair [21]. A Swedish registry study likewise demonstrated on comparing anterior mesh repair with laparo-endoscopic mesh repair for recurrent hernias a lower risk of chronic pain for the laparo-endoscopic operation (OR 0.54 [CI 0.30-0.97]; $p=0.039$ ) [22].

On the basis of the meta-analyses, the European Hernia Society recommends laparo-endoscopic inguinal hernia repair of recurrent hernias after conventional open repair $[8,23]$ and for recurrent hernias after laparo-endoscopic hernia repair an open procedure. Likewise, the International Endohernia Society recommends, with a high level of evidence, TEP and TAPP for repair of recurrent hernia as the preferred alternative to tissue repair and to the Lichtenstein repair after prior anterior repair [24, 25]. In the Consensus Development Conference of the European Association of Endoscopic Surgery, TEP and TAPP are preferred in patients with a recurrent groin hernia after open repair. Repeat endoscopic repair is only feasible when the surgeon has a high level of experience in repeat endoscopic groin hernia repair [26]. However, registry data show that even following previous open suture and mesh repair to treat the primary inguinal hernia, open suture and mesh repair are used once again for a recurrent hernia [27]. That is due to the fact that the skill needed for laparoendoscopic recurrent inguinal hernia repairs was not always assured. Where surgeons had used an open technique to repair $95 \%$ of primary inguinal hernias, then more than $90 \%$ of recurrences were also repaired using an open procedure [28]. That was also true when using mesh repair for the primary inguinal hernia operation [13].

This present analysis of data from the Herniamed Hernia Registry [29] now investigates: (1) To what extent surgeons implement the guidelines of the international hernia societies. (2) Since to date no study has compared the outcomes of open and laparo-endoscopic recurrent inguinal hernia repair carried out in compliance with the guidelines, that aspect will now also be explored in the present analysis. (3) Finally, how the outcomes of open and laparoendoscopic recurrent inguinal hernia repair differ on compliance versus non-compliance with the guidelines.

\section{Patients and methods}

The Herniamed Registry is a multicenter, Internet-based hernia registry [29] into which 427 participating hospitals and surgeons engaged in private practice (Herniamed Study Group) have entered data prospectively on their patients who had undergone routine hernia surgery and signed an informed consent to participate. All postoperative complications occurring up to 30 days after surgery are recorded. On 1-year follow-up, postoperative complications are once again reviewed when the general practitioner and patient complete a questionnaire. Information is also obtained on any recurrence, pain at rest and on exertion as well as pain requiring treatment. This present analysis compares the prospective data collected for all male patients with a minimum age of 16 years who had undergone elective recurrent unilateral inguinal hernia repair using either transabdominal preperitoneal patch plasty (TAPP), total extraperitoneal patch plasty (TEP) or open repair in Lichtenstein, Should ice, TIPP and Plug techniques.

In total, 4812 patients were enrolled between September 1, 2009, and August 31, 2013 (Fig. 1). Of these patients, 2482 (51.58\%) had laparo-endoscopic and 2330 (48.42\%) open repair. All the patients had to have a 1-year follow-up (follow-up rate $100 \%$ ).

The demographic and surgery-related parameters included age (years), BMI $\left(\mathrm{kg} / \mathrm{m}^{2}\right)$, ASA classification (I, II, III-IV) as well as EHS classification (hernia type: medial, lateral, femoral, scrotal and defect size: grade $\mathrm{I}=<1.5 \mathrm{~cm}$, grade $\mathrm{II}=1.5-3 \mathrm{~cm}$, grade $\mathrm{III}=>3 \mathrm{~cm}$ ) [30] and general risk factors (nicotine, COPD, diabetes, cortisone, immunosuppression, etc.). Risk factors were dichotomized, i.e., 'yes' if at least one risk factor is positive and 'no' otherwise.

The dependent variables were intra- and postoperative complication rates, number of reoperations due to complications as well as the 1-year results (recurrence rate, pain at rest, pain on exertion and pain requiring treatment). 
Fig. 1 Flowchart of patient inclusion

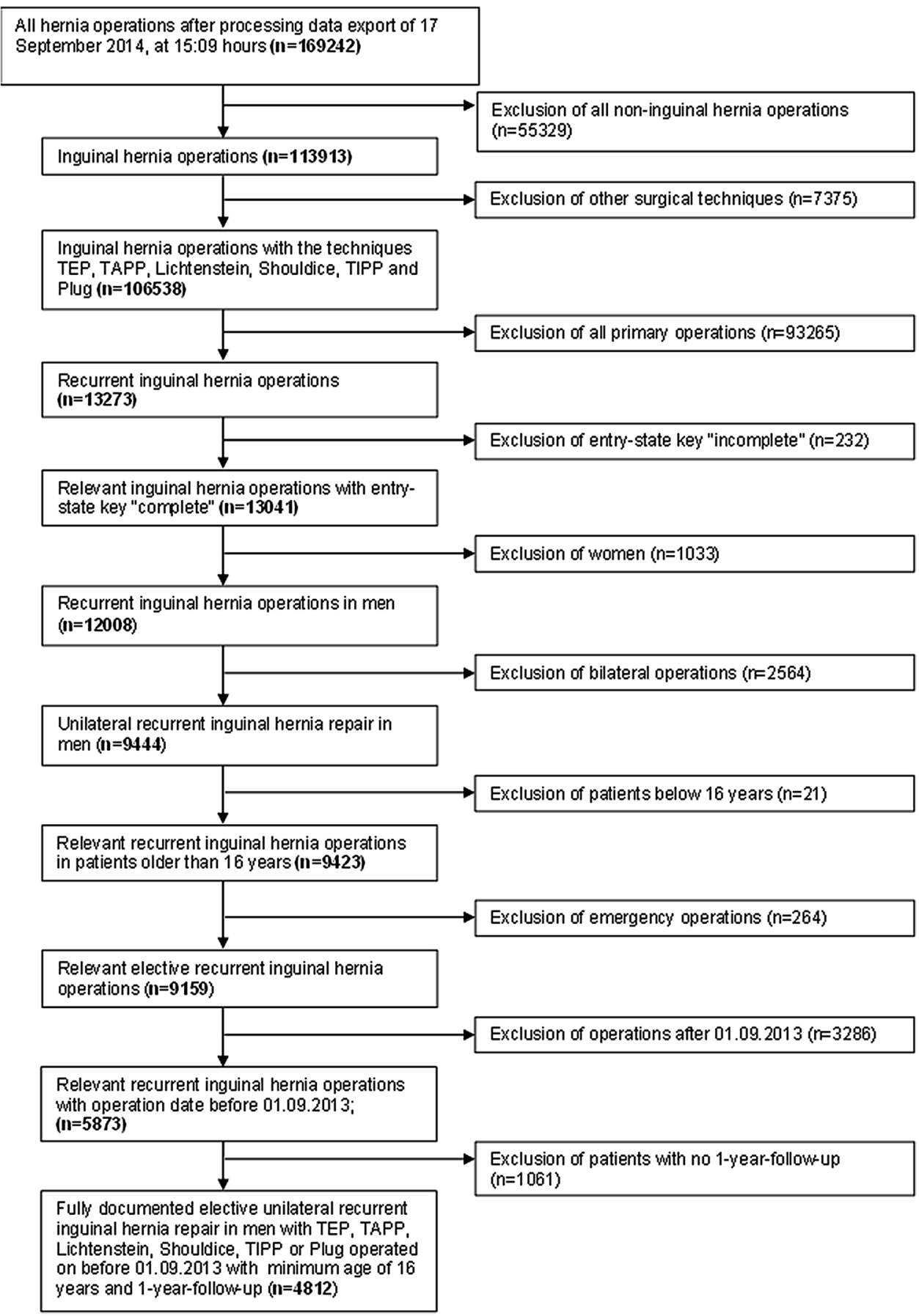

All analyses were performed with the software 9.2 (SAS 9.2 Institute Inc. Cary, NY, USA) and intentionally calculated to a full significance level of $5 \%$, i.e., they were not corrected in respect of multiple tests, and each $p$ value $\leq 0.05$ represents a significant result. To discern differences between the groups in unadjusted analyses, Fisher's exact test was used for categorical outcome variables and the robust t-test (Satterthwaite) for continuous variables.

To rule out any confounding of data caused by different patient characteristics, the results of unadjusted analyses were verified via multivariable analyses in which, in addition to laparo-endoscopic or open operation, other influence parameters were simultaneously reviewed.

To identify influence factors in multivariable analyses, the binary logistic regression model for dichotomous outcome variables was used. Estimates for odds ratio (OR) and the corresponding 95\% confidence interval based on the Wald test were given. For influence variables with more than two categories, one of the latter forms was used in each case as reference category. For age (years) the 10-year 
OR estimate and for BMI $\left(\mathrm{kg} / \mathrm{m}^{2}\right)$ the five-point OR estimate were given. Results were presented in tabular form, sorted by descending impact.

\section{Results}

1. To what extent do surgeons follow the guidelines?

In the laparo-endoscopic recurrent operation group, the recurrent operation was performed for $n=1528 / 2482$ (61.6\%) patients following the open suture technique for $n=718 / 2482(28.9 \%)$ after open mesh repair, and for $n=233 / 2482$ (9.4\%) following laparo-endoscopic primary mesh repair (unknown $0.1 \%$ ).

Open recurrent repair was performed for $n=1011 / 2330$ (43.4\%) patients following previous open suture repair, for $n=897 / 2330$ (38.5\%) patients following laparo-endoscopic mesh repair and for 412/2330 (17.7\%) patients after open mesh repair of the primary inguinal hernia (unknown $0.4 \%$ ).

Accordingly, in the laparo-endoscopic recurrent repair group $90.5 \%$, and in the open recurrent repair group 38.5\%, of patients were operated on in compliance with the guidelines of the international hernia societies.

2. Is there a difference in the outcome of open versus laparo-endoscopic recurrent inguinal hernia repair in compliance with the guidelines?

This analysis is based on $n=2246$ laparo-endoscopic recurrent inguinal hernia repair operations following previous open primary operation and $n=897$ open recurrent inguinal hernia repair operations following previous laparo-endoscopic primary repair (Table 1). Unadjusted analysis did not find any significant difference in the mean age between the two groups; however, the mean BMI value was higher for those patients undergoing open recurrent repair (Table 2). The open recurrent repair was associated with significantly larger hernia defects, more medial, fewer femoral and lateral EHS classifications (Table 3). No differences were identified in the risk factors (Table 3). Nonadjusted analysis of the target variables revealed that the intraoperative complications entailed more nerve injuries for open recurrent repair as well as more pain at rest and
Table 2 Age and BMI of patients with laparo-endoscopic versus open unilateral recurrent inguinal hernia repair in men according to the guidelines

\begin{tabular}{lllll}
\hline & & \multicolumn{2}{l}{ Operation } & \multirow{2}{*}{$p$} \\
\cline { 3 - 4 } & & Endoscopic & Open & \\
\hline Age (years) & Mean \pm STD & $58.9 \pm 15.6$ & $59.3 \pm 15.3$ & 0.440 \\
BMI $\left(\mathrm{kg} / \mathrm{m}^{2}\right)$ & Mean \pm STD & $25.9 \pm 3.4$ & $26.3 \pm 3.6$ & 0.004 \\
\hline
\end{tabular}

pain on exertion on 1-year follow-up (Table 4). No significant difference was detected between the laparo-endoscopic and open technique on performing recurrent repair in compliance with the guidelines for the following: overall intraoperative complication rate, postoperative complication rate, complication-related reoperation rate, recurrence rate and the rate of chronic pain requiring treatment.

For multivariable analysis of intraoperative complications, complication-related reoperations and recurrence on 1-year follow-up, it was not possible to calculate any model because of the paucity of relevant cases. The results of the model that explored the variables influencing onset of postoperative complications are illustrated in Table 5 (model matching: $p=0.002$ ). Only medial EHS localization impacted the postoperative complication rate. Medial EHS classification reduced the risk of postoperative complications (OR 0.427 [0.213; 0.857]; $p=0.017$ ). But there was no evidence of the surgical technique having impacted the postoperative complication rate. The multivariable analysis results of pain at rest are presented in Table 6 (model matching: $p<0.001$ ). Here, the BMI proved to be the strongest influence factor $(p=0.001)$. A five-point higher BMI increased the risk of pain at rest (five-point OR $1.351[1.127 ; 1.620])$. On the other hand, laparo-endoscopic operation (OR 0.643 [0.476; 0.868]; $p=0.004$ ) and larger defect size (III vs I: OR 0.500 [0.307; 0.815]; $p=0.021)$ significantly reduced the risk of pain at rest. The multivariable analysis results of pain on exertion are given in Table 7 (model matching: $p<0.001$ ). These were highly significantly affected by age and hernia defect size $(p<0.001)$. A higher age $(10$-year OR 0.825 [0.760; 0.897]) as well as larger hernias (II vs I: OR 0.704 [0.541;
Table 1 Recurrent operations according to the guidelines and previous operations

\begin{tabular}{|c|c|c|c|c|c|c|c|c|c|c|}
\hline & \multicolumn{8}{|c|}{ Previous operations } & \multirow{2}{*}{\multicolumn{2}{|c|}{ Total }} \\
\hline & \multicolumn{2}{|c|}{ Unknown } & \multicolumn{2}{|c|}{ Suture } & \multicolumn{2}{|c|}{ Open mesh } & \multicolumn{2}{|c|}{ Endoscopic mesh } & & \\
\hline & $N$ & $\%$ & $N$ & $\%$ & $N$ & $\%$ & $N$ & $\%$ & $N$ & $\%$ \\
\hline \multicolumn{11}{|c|}{ Recurrent operation } \\
\hline Endoscopic & 3 & 0.1 & 1528 & 61.6 & 718 & 28.9 & 233 & 9.4 & 2482 & 100.0 \\
\hline Open & 10 & 0.4 & 1011 & 43.4 & 412 & 17.7 & 897 & 38.5 & 2330 & 100.0 \\
\hline Total & 13 & 0.3 & 2539 & 52.8 & 1130 & 23.5 & 1130 & 23.5 & 4812 & 100.0 \\
\hline
\end{tabular}

Bold numbers are the operations in accordance with the guidelines 
Table 3 Demographic and surgery-related parameters and risk factors for patients with laparo-endoscopic versus open unilateral recurrent inguinal hernia repair in men according to the guidelines

\begin{tabular}{|c|c|c|c|c|c|c|}
\hline & & \multicolumn{2}{|c|}{ Endoscopic } & \multicolumn{2}{|c|}{ Open } & \multirow[t]{2}{*}{$p$} \\
\hline & & $n$ & $\%$ & $n$ & $\%$ & \\
\hline \multirow[t]{3}{*}{ ASA score } & I & 561 & 24.98 & 257 & 28.65 & \multirow[t]{3}{*}{0.091} \\
\hline & II & 1302 & 57.97 & 502 & 55.96 & \\
\hline & III/IV & 383 & 17.05 & 138 & 15.38 & \\
\hline \multirow[t]{3}{*}{ Defect size } & $\mathrm{I}(<1.5 \mathrm{~cm})$ & 417 & 18.57 & 151 & 16.83 & \multirow[t]{3}{*}{$<0.001$} \\
\hline & II $(1.5-3 \mathrm{~cm})$ & 1459 & 64.96 & 493 & 54.96 & \\
\hline & III $(>3 \mathrm{~cm})$ & 370 & 16.47 & 253 & 28.21 & \\
\hline \multirow[t]{2}{*}{ EHS-classification medial } & Yes & 1112 & 49.51 & 518 & 57.75 & \multirow[t]{2}{*}{$<0.001$} \\
\hline & No & 1134 & 50.49 & 379 & 42.25 & \\
\hline \multirow[t]{2}{*}{ EHS-classification lateral } & Yes & 1351 & 60.15 & 452 & 50.39 & \multirow[t]{2}{*}{$<0.001$} \\
\hline & No & 895 & 39.85 & 445 & 49.61 & \\
\hline \multirow[t]{2}{*}{ EHS-classification femoral } & Yes & 77 & 3.43 & 15 & 1.67 & \multirow[t]{2}{*}{0.007} \\
\hline & No & 2169 & 96.57 & 882 & 98.33 & \\
\hline \multirow[t]{2}{*}{ EHS-classification scrotal } & Yes & 27 & 1.20 & 12 & 1.34 & \multirow[t]{2}{*}{0.724} \\
\hline & No & 2219 & 98.80 & 885 & 98.66 & \\
\hline \multicolumn{7}{|l|}{ Risk factor } \\
\hline \multirow[t]{2}{*}{ Total } & Yes & 687 & 30.59 & 275 & 30.66 & \multirow[t]{2}{*}{0.966} \\
\hline & No & 1559 & 69.41 & 622 & 69.34 & \\
\hline \multirow[t]{2}{*}{ COPD } & Yes & 151 & 6.72 & 66 & 7.36 & \multirow[t]{2}{*}{0.534} \\
\hline & No & 2095 & 93.28 & 831 & 92.64 & \\
\hline \multirow[t]{2}{*}{ Diabetes } & Yes & 129 & 5.74 & 51 & 5.69 & \multirow[t]{2}{*}{1.000} \\
\hline & No & 2117 & 94.26 & 846 & 94.31 & \\
\hline \multirow[t]{2}{*}{ Aortic aneurism } & Yes & 16 & 0.71 & 4 & 0.45 & \multirow[t]{2}{*}{0.467} \\
\hline & No & 2230 & 99.29 & 893 & 99.55 & \\
\hline \multirow[t]{2}{*}{ Immunosuppression } & Yes & 14 & 0.62 & 10 & 1.11 & \multirow[t]{2}{*}{0.174} \\
\hline & No & 2232 & 99.38 & 887 & 98.89 & \\
\hline \multirow[t]{2}{*}{ Corticoids } & Yes & 20 & 0.89 & 8 & 0.89 & 1.000 \\
\hline & No & 2226 & 99.11 & 889 & 99.11 & \\
\hline Smoking & Yes & 262 & 11.67 & 110 & 12.26 & 0.669 \\
\hline & No & 1984 & 88.33 & 787 & 87.74 & \\
\hline Coagulopathy & Yes & 33 & 1.47 & 9 & 1.00 & 0.390 \\
\hline & No & 2213 & 98.53 & 888 & 99.00 & \\
\hline Antiplatelet medication & Yes & 202 & 8.99 & 79 & 8.81 & 0.890 \\
\hline & No & 2044 & 91.01 & 818 & 91.19 & \\
\hline Anticoagulation therapy & Yes & 44 & 1.96 & 25 & 2.79 & 0.177 \\
\hline & No & 2202 & 98.04 & 872 & 97.21 & \\
\hline
\end{tabular}

0.916]; III vs I: OR 0.479 [0.331; 0.693]) reduced the risk of pain on exertion. Likewise, laparo-endoscopic operations (OR 0.679 [0.537; 0.857]; $p=0.001$ ) compared with open operations reduced the risk for onset of pain on exertion. Similarly, lateral EHS classification reduced the risk (OR 0.624 [0.422; 0.922]; $p=0.018$ ) of pain on exertion. However, the risk was increased in association with a five-point higher BMI (five-point OR 1.251 [1.081; 1.449]; $p=0.003$ ). The multivariable analysis results of chronic pain requiring treatment are presented in Table 8 (model matching: $p=0.005$ ). Here, only the BMI proved to be a significant influence factor $(p=0.014)$. A five- point higher $\mathrm{BM}$ increased the rate of pain requiring treatment (five-point OR 1.320 [1.058; 1.647]). However, there was no evidence of the surgical technique having impacted the rate of pain requiring treatment.

3a. How do the outcomes of laparo-endoscopic recurrent inguinal hernia repair differ on compliance versus non-compliance with the guidelines?

In the laparo-endoscopic recurrent operation group, the recurrent operation was performed for $n=233 / 2482$ (9.4\%) patients following laparo-endoscopic primary mesh repair, i.e., not in compliance with the guidelines of the 
Table 4 Intra- and postoperative complications, complication-related reoperations and 1-year followup results of patients with laparo-endoscopic versus open unilateral recurrent inguinal hernia repair in men according to the guidelines

\begin{tabular}{|c|c|c|c|c|c|c|}
\hline & & \multicolumn{2}{|c|}{ Endoscopic } & \multicolumn{2}{|c|}{ Open } & \multirow[t]{2}{*}{$p$} \\
\hline & & $n$ & $\%$ & $n$ & $\%$ & \\
\hline \multicolumn{7}{|l|}{ Intraoperative complication } \\
\hline \multirow[t]{2}{*}{ Total } & Yes & 26 & 1.16 & 14 & 1.56 & 0.380 \\
\hline & No & 2220 & 98.84 & 883 & 98.44 & \\
\hline \multirow[t]{2}{*}{ Bleeding } & Yes & 15 & 0.67 & 3 & 0.33 & 0.431 \\
\hline & No & 2231 & 99.33 & 894 & 99.67 & \\
\hline \multicolumn{7}{|l|}{ Injuries } \\
\hline \multirow[t]{2}{*}{ Total } & Yes & 17 & 0.76 & 12 & 1.34 & 0.147 \\
\hline & No & 2229 & 99.24 & 885 & 98.66 & \\
\hline \multirow[t]{2}{*}{ Vascular } & Yes & 8 & 0.36 & 0 & 0.00 & 0.115 \\
\hline & No & 2238 & 99.64 & 897 & 100.0 & \\
\hline \multirow[t]{2}{*}{ Bowel } & Yes & 5 & 0.22 & 0 & 0.00 & 0.330 \\
\hline & No & 2241 & 99.78 & 897 & 100.0 & \\
\hline \multirow[t]{2}{*}{ Bladder } & Yes & 2 & 0.09 & 1 & 0.11 & 1.000 \\
\hline & No & 2244 & 99.91 & 896 & 99.89 & \\
\hline \multirow[t]{2}{*}{ Nerve } & Yes & 0 & 0.00 & 9 & 1.00 & $<0.001$ \\
\hline & No & 2246 & 100.0 & 888 & 99.00 & \\
\hline \multicolumn{7}{|l|}{ Postoperative complication } \\
\hline \multirow[t]{2}{*}{ Total } & Yes & 80 & 3.56 & 33 & 3.68 & 0.916 \\
\hline & No & 2166 & 96.44 & 864 & 96.32 & \\
\hline \multirow[t]{2}{*}{ Bleeding } & Yes & 29 & 1.29 & 17 & 1.90 & 0.248 \\
\hline & No & 2217 & 98.71 & 880 & 98.10 & \\
\hline \multirow[t]{2}{*}{ Seroma } & Yes & 51 & 2.27 & 14 & 1.56 & 0.266 \\
\hline & No & 2195 & 97.73 & 883 & 98.44 & \\
\hline \multirow[t]{2}{*}{ Bowell injury/anastomotic leakage } & Yes & 1 & 0.04 & 0 & 0.00 & 1.000 \\
\hline & No & 2245 & 99.96 & 897 & 100.0 & \\
\hline \multirow[t]{2}{*}{ Wound healing disorders } & Yes & 2 & 0.09 & 4 & 0.45 & 0.059 \\
\hline & No & 2244 & 99.91 & 893 & 99.55 & \\
\hline \multirow[t]{2}{*}{ Ileus } & - & - & - & - & - & - \\
\hline & No & 2246 & 100.0 & 897 & 100.0 & \\
\hline \multirow[t]{2}{*}{ Reoperations } & Yes & 27 & 1.20 & 9 & 1.00 & 0.714 \\
\hline & No & 2219 & 98.80 & 888 & 99.00 & \\
\hline \multirow[t]{2}{*}{ Recurrence on follow-up } & Yes & 28 & 1.25 & 10 & 1.11 & 0.858 \\
\hline & No & 2218 & 98.75 & 887 & 98.89 & \\
\hline \multirow[t]{2}{*}{ Pain in rest on follow-up } & Yes & 133 & 5.92 & 78 & 8.70 & 0.007 \\
\hline & No & 2113 & 94.08 & 819 & 91.30 & \\
\hline \multirow[t]{2}{*}{ Pain on exertion on follow-up } & Yes & 250 & 11.13 & 135 & 15.05 & 0.003 \\
\hline & No & 1996 & 88.87 & 762 & 84.95 & \\
\hline \multirow[t]{2}{*}{ Pain requiring treatment } & Yes & 85 & 3.78 & 40 & 4.46 & 0.419 \\
\hline & No & 2161 & 96.22 & 857 & 95.54 & \\
\hline
\end{tabular}

conducted in compliance with the guidelines revealed a significantly higher proportion of larger defects as well as a smaller proportion of lateral inguinal hernia recurrences (Table 11). No relevant differences were found for the other variables and risk factors. When recurrent repair was performed as per the guidelines, the laparo-endoscopic procedure was found to be associated with fewer intraoperative (1.2 vs $3.0 \% ; p=0.019)$ and postoperative (Table 10). The laparo-endoscopic recurrent repairs not 
Table 5 Multivariable analysis of postoperative complications in patients with recurrent inguinal hernia repair according to the guidelines
Table 6 Multivariable analysis of pain in rest in 1-year followup in patients with recurrent inguinal hernia repair according to the guidelines

\begin{tabular}{llllll}
\hline Parameter & $p$ value & Category & OR estimate & \multicolumn{2}{l}{$95 \%$ CI } \\
\hline EHS-classification medial & 0.017 & Yes versus no & 0.427 & 0.213 & 0.857 \\
Age $(10$-year OR) & 0.081 & & 1.148 & 0.983 & 1.339 \\
Defect size & 0.118 & II $(1.5-3 \mathrm{~cm})$ versus I $(<1.5 \mathrm{~cm})$ & 0.848 & 0.502 & 1.434 \\
& & III $(>3 \mathrm{~cm})$ versus I $(<1.5 \mathrm{~cm})$ & 1.382 & 0.756 & 2.526 \\
Risk factors & 0.139 & Yes versus no & 1.371 & 0.903 & 2.083 \\
BMI (five-point OR) & 0.155 & & 0.807 & 0.600 & 1.085 \\
ASA score & 0.306 & II versus I & 0.817 & 0.486 & 1.370 \\
& & III/IV versus I & 1.177 & 0.600 & 2.308 \\
EHS-classification lateral & 0.372 & Yes versus no & 0.723 & 0.354 & 1.474 \\
EHS-classification femoral & 0.647 & Yes versus no & 1.263 & 0.466 & 3.426 \\
Operation & 0.772 & Endoscopic versus open & 0.939 & 0.616 & 1.434 \\
EHS-classification scrotal & 0.862 & Yes versus no & 1.121 & 0.308 & 4.077 \\
\hline
\end{tabular}

\begin{tabular}{llllll}
\hline Parameter & $p$ value & Category & OR estimate & \multicolumn{2}{l}{$95 \%$ CI } \\
\hline BMI (five-point OR) & 0.001 & & 1.351 & 1.127 & 1.620 \\
Operation & 0.004 & Endoscopic versus open & 0.643 & 0.476 & 0.868 \\
Defect size & 0.021 & II $(1.5-3 \mathrm{~cm})$ versus I $(<1.5 \mathrm{~cm})$ & 0.794 & 0.562 & 1.123 \\
& & III $(>3 \mathrm{~cm})$ versus I $(<1.5 \mathrm{~cm})$ & 0.500 & 0.307 & 0.815 \\
Age (10-year OR) & 0.064 & & 0.902 & 0.809 & 1.006 \\
EHS-classification lateral & 0.087 & Yes versus no & 0.629 & 0.370 & 1.070 \\
EHS-classification medial & 0.122 & Yes versus no & 0.659 & 0.389 & 1.118 \\
Risk factor & 0.129 & Yes versus no & 1.278 & 0.931 & 1.754 \\
EHS-classification femoral & 0.834 & Yes versus no & 0.913 & 0.392 & 2.130 \\
ASA score & 0.888 & II versus I & 0.917 & 0.643 & 1.307 \\
& & III/IV versus I & 0.943 & 0.552 & 1.610 \\
EHS-classification scrotal & 0.974 & Yes versus no & 0.000 & 0.000 & I \\
\hline
\end{tabular}

I Infinity

\begin{tabular}{lcllll}
\hline Parameter & $p$ value & Category & OR estimate & \multicolumn{2}{l}{$95 \%$ CI } \\
\hline Age (10-year OR) & $<0.001$ & & 0.825 & 0.760 & 0.897 \\
Defect size & $<0.001$ & II $(1.5-3 \mathrm{~cm})$ versus I $(<1.5 \mathrm{~cm})$ & 0.704 & 0.541 & 0.916 \\
& & III $(>3 \mathrm{~cm})$ versus I $(<1.5 \mathrm{~cm})$ & 0.479 & 0.331 & 0.693 \\
Operation & 0.001 & Endoscopic versus open & 0.679 & 0.537 & 0.857 \\
BMI (five-point OR) & 0.003 & & 1.251 & 1.081 & 1.449 \\
EHS-classification lateral & 0.018 & Yes versus no & 0.624 & 0.422 & 0.922 \\
EHS-classification scrotal & 0.094 & Yes versus no & 0.178 & 0.024 & 1.339 \\
EHS-classification medial & 0.180 & Yes versus no & 0.765 & 0.517 & 1.131 \\
Risk factor & 0.512 & Yes versus no & 1.087 & 0.847 & 1.393 \\
ASA score & 0.764 & II versus I & 0.981 & 0.749 & 1.285 \\
& & III/IV versus I & 1.114 & 0.737 & 1.682 \\
EHS-classification femoral & 0.933 & Yes versus no & 0.973 & 0.511 & 1.850 \\
\hline
\end{tabular}

Table 7 Multivariable analysis of pain on exertion in 1-year follow-up in patients with recurrent inguinal hernia repair according to the guidelines complications (3.6 vs $8.6 \% ; p<0.001$ ) as well as a lower re-recurrence risk ( 1.2 vs $3.4 \% ; p=0.008$; Table 12 ). No differences were identified for the pain rates.
For multivariable analysis of the intraoperative complications, complication-related reoperations and re-recurrences, it was not possible to calculate a valid model on 
Table 8 Multivariable analysis of chronic pain requiring treatment in 1-year follow-up in patients with recurrent inguinal hernia repair according to the guidelines
Table 9 Laparo-endoscopic unilateral recurrent inguinal hernia repairs on compliance versus non-compliance with the guidelines

\begin{tabular}{llllll}
\hline Parameter & $p$ value & Category & OR estimate & 95\% CI \\
\hline BMI (five-point OR) & 0.014 & & 1.320 & 1.058 & 1.647 \\
EHS-classification lateral & 0.051 & Yes versus no & 0.494 & 0.243 & 1.004 \\
Age (10-year OR) & 0.053 & & 0.871 & 0.758 & 1.002 \\
EHS-classification medial & 0.054 & Yes versus no & 0.501 & 0.248 & 1.012 \\
ASA score & 0.240 & II versus I & 1.048 & 0.654 & 1.679 \\
& & III/IV versus I & 1.607 & 0.834 & 3.094 \\
Risk factor & 0.253 & Yes versus no & 1.263 & 0.846 & 1.886 \\
Operation & 0.260 & Endoscopic versus open & 0.797 & 0.538 & 1.182 \\
Defect size & 0.294 & II $(1.5-3$ cm) versus I $(<1.5 \mathrm{~cm})$ & 0.944 & 0.597 & 1.493 \\
& & III $(>3$ cm) versus I $(<1.5 \mathrm{~cm})$ & 0.634 & 0.338 & 1.191 \\
EHS-classification femoral & 0.476 & Yes versus no & 1.390 & 0.561 & 3.445 \\
EHS-classification scrotal & 0.979 & Yes versus no & 0.000 & 0.000 & I \\
\hline
\end{tabular}

I Infinity

\begin{tabular}{|c|c|c|c|c|c|c|c|c|}
\hline & \multicolumn{6}{|c|}{ Previous operations } & \multirow{2}{*}{\multicolumn{2}{|c|}{ Total }} \\
\hline & \multicolumn{2}{|c|}{ Suture } & \multicolumn{2}{|c|}{ Open mesh } & \multicolumn{2}{|c|}{ Endoscopic mesh } & & \\
\hline & $N$ & ColPctN & $N$ & ColPctN & $N$ & ColPctN & $N$ & ColPctN \\
\hline \multicolumn{9}{|c|}{ Guidelines } \\
\hline No & - & - & - & - & 233 & 100.0 & 233 & 9.4 \\
\hline Yes & 1528 & 100.0 & 718 & 100.0 & - & - & 2246 & 90.6 \\
\hline Total & 1528 & 100.0 & 718 & 100.0 & 233 & 100.0 & 2479 & 100.0 \\
\hline
\end{tabular}

Table 10 Age and BMI of patients with laparo-endoscopic unilateral recurrent inguinal hernia repair on compliance versus non-compliance with the guidelines

\begin{tabular}{lllll}
\hline & & \multicolumn{2}{l}{ Guidelines } & \multirow{2}{*}{$p$} \\
\cline { 3 - 4 } & & Yes & No & \\
\hline \multirow{2}{*}{ Age (years) } & Mean \pm STD & $58.9 \pm 15.6$ & $60.1 \pm 14.2$ & 0.199 \\
BMI & Mean \pm STD & $25.9 \pm 3.4$ & $26.2 \pm 3.0$ & 0.306 \\
\hline
\end{tabular}

differences of follow-up because of the small number of positive cases. On univariable analysis of pain at rest, pain on exertion and chronic pain requiring treatment, no difference was discerned for the procedures conducted in accordance with the guidelines.

The multivariable analysis results for the postoperative complications are presented in Table 13 (model matching: $p<0.001$ ). The postoperative complications were impacted, in particular, by the procedures conducted in accordance with the guidelines $(p=0.001)$. When the guidelines were observed, the risk of onset of postoperative complications declined (OR 0.419 [0.248; 0.708]; $p=0.001)$. Besides, the defect size had a significant effect on the postoperative complication risk. Larger hernia defects (III vs I: OR 2.329 [1.135; 4.779]; $p=0.018$ ) were associated with a higher complication risk.

$3 \mathrm{~b}$. How do the outcomes of open recurrent inguinal hernia repair differ on compliance versus non-compliance with the guidelines?

In the open recurrent repair group, only $n=897 / 2.320$ (38.5\%) of operations were performed following previous primary laparo-endoscopic inguinal hernia repair, i.e., according to the guidelines. Conduct of open recurrent repair following previous suture procedure for the primary inguinal hernia repair $(n=1.011 / 2.320 ; 43.4 \%)$ and after mesh procedure $(n=412 / 2.320 ; 17.7 \%)$ was not in compliance with the guidelines (Table 14). Below are now compared the open recurrent inguinal hernia repair procedures conducted on compliance $(n=897 / 2.320 ; 38.5 \%)$ versus non-compliance with the guidelines $(n=1.423$ / 2.320; 61.3\%).

Patients with recurrent inguinal hernias repaired in accordance with the guidelines had a significantly lower age and higher BMI (Table 15). Furthermore, patients operated on with an open procedure as per the guidelines had a significantly lower ASA score, smaller hernia defects, fewer risk factors and fewer lateral and scrotal hernias (Table 16). When the recurrent repair was 
Table 11 Demographic and surgery-related parameters and risk factors for patients with laparo-endoscopic unilateral recurrent inguinal hernia repair on compliance versus noncompliance with the guidelines

\begin{tabular}{|c|c|c|c|c|c|c|}
\hline & & \multicolumn{4}{|c|}{ Guideline } & \multirow[t]{3}{*}{$p$} \\
\hline & & \multicolumn{2}{|l|}{ Yes } & \multicolumn{2}{|l|}{ No } & \\
\hline & & $n$ & $\%$ & $n$ & $\%$ & \\
\hline \multirow[t]{3}{*}{ ASA score } & I & 562 & 24.99 & 59 & 25.32 & \multirow[t]{3}{*}{0.992} \\
\hline & II & 1303 & 57.94 & 134 & 57.51 & \\
\hline & III/IV & 384 & 17.07 & 40 & 17.17 & \\
\hline \multirow[t]{3}{*}{ Defect size } & $\mathrm{I}(<1.5 \mathrm{~cm})$ & 419 & 18.63 & 34 & 14.59 & \multirow[t]{3}{*}{0.001} \\
\hline & II $(1.5-3 \mathrm{~cm})$ & 1460 & 64.92 & 139 & 59.66 & \\
\hline & III $(>3 \mathrm{~cm})$ & 370 & 16.45 & 60 & 25.75 & \\
\hline \multicolumn{7}{|l|}{ Risk factor } \\
\hline \multirow[t]{2}{*}{ Total } & Yes & 687 & 30.55 & 60 & 25.75 & \multirow[t]{2}{*}{0.129} \\
\hline & No & 1562 & 69.45 & 173 & 74.25 & \\
\hline \multirow[t]{2}{*}{ COPD } & Yes & 151 & 6.71 & 14 & 6.01 & \multirow[t]{2}{*}{0.681} \\
\hline & No & 2098 & 93.29 & 219 & 93.99 & \\
\hline \multirow[t]{2}{*}{ Diabetes } & Yes & 129 & 5.74 & 10 & 4.29 & \multirow[t]{2}{*}{0.361} \\
\hline & No & 2120 & 94.26 & 223 & 95.71 & \\
\hline \multirow[t]{2}{*}{ Aortic aneurism } & Yes & 16 & 0.71 & 1 & 0.43 & \multirow[t]{2}{*}{0.619} \\
\hline & No & 2233 & 99.29 & 232 & 99.57 & \\
\hline \multirow[t]{2}{*}{ Immunosuppression } & Yes & 14 & 0.62 & 1 & 0.43 & \multirow[t]{2}{*}{0.717} \\
\hline & No & 2235 & 99.38 & 232 & 99.57 & \\
\hline \multirow{2}{*}{ Corticoids } & Yes & 20 & 0.89 & 1 & 0.43 & \multirow[t]{2}{*}{0.465} \\
\hline & No & 2229 & 99.11 & 232 & 99.57 & \\
\hline \multirow[t]{2}{*}{ Smoking } & Yes & 262 & 11.65 & 30 & 12.88 & \multirow[t]{2}{*}{0.580} \\
\hline & No & 1987 & 88.35 & 203 & 87.12 & \\
\hline \multirow[t]{2}{*}{ Coagulopathy } & Yes & 33 & 1.47 & 3 & 1.29 & \multirow[t]{2}{*}{0.827} \\
\hline & No & 2216 & 98.53 & 230 & 98.71 & \\
\hline \multirow[t]{2}{*}{ Antiplatelet medication } & Yes & 202 & 8.98 & 15 & 6.44 & \multirow[t]{2}{*}{0.191} \\
\hline & No & 2047 & 91.02 & 218 & 93.56 & \\
\hline Anticoagulation therapy & Yes & 44 & 1.96 & 4 & 1.72 & 0.800 \\
\hline & No & 2205 & 98.04 & 229 & 98.28 & \\
\hline EHS-classification medial & Yes & 1115 & 49.58 & 120 & 51.50 & 0.576 \\
\hline & No & 1134 & 50.42 & 113 & 48.50 & \\
\hline EHS-classification lateral & Yes & 1351 & 60.07 & 118 & 50.64 & 0.005 \\
\hline & No & 898 & 39.93 & 115 & 49.36 & \\
\hline EHS-classification femoral & Yes & 77 & 3.42 & 6 & 2.58 & 0.493 \\
\hline & No & 2172 & 96.58 & 227 & 97.42 & \\
\hline EHS-classification scrotal & Yes & 27 & 1.20 & 5 & 2.15 & 0.223 \\
\hline & No & 2222 & 98.80 & 228 & 97.85 & \\
\hline
\end{tabular}

performed as per the guidelines, open repair was associated with fewer postoperative complications (3.6 vs $5.8 \%$; $p=0.021)$ and complication-related reoperation (1.0 vs $2.1 \% ; p=0.041)$ as well as a lower re-recurrence risk (1.1 vs $2.6 \% ; p=0.012)$. On the other hand, there was an increase in the risk of pain at rest ( 8.6 vs $5.4 \% ; p=0.003$ ) and on exertion (15.0 vs $10.2 \% ; p<0.001$; Table 17$)$.

For multivariable analysis of the intraoperative complications, complication-related reoperations and re-recurrences, it was not possible to calculate a valid model since the number of positive cases was too small. Univariable analysis of chronic pain requiring treatment did not detect any difference for repair as per the guidelines; therefore, no multivariable model was calculated.

The multivariable analysis results of variables influencing onset of postoperative complications are given in Table 18 (model matching: $p=0.002$ ).

The postoperative complications were only affected by age, with older patients (10-year OR 1.275 [1.085; 1.498]; $p=0.003$ ) having a higher risk of postoperative complications. There was no evidence that repair as per the guidelines impacted the postoperative complications. 
Table 12 Intra- and postoperative compilations, complication-related reoperations and 1-year followup-results of patients with laparo-endoscopic unilateral recurrent inguinal hernia repair on compliance versus noncompliance with the guidelines

\begin{tabular}{|c|c|c|c|c|c|c|}
\hline & & \multicolumn{4}{|c|}{ Guidelines } & \multirow[t]{3}{*}{$p$} \\
\hline & & \multicolumn{2}{|l|}{ Yes } & \multicolumn{2}{|l|}{ No } & \\
\hline & & $n$ & $\%$ & $n$ & $\%$ & \\
\hline \multicolumn{7}{|l|}{ Intraoperative complication } \\
\hline \multirow[t]{2}{*}{ Total } & Yes & 26 & 1.16 & 7 & 3.00 & 0.019 \\
\hline & No & 2223 & 98.84 & 226 & 97.00 & \\
\hline \multirow[t]{2}{*}{ Bleeding } & Yes & 15 & 0.67 & 7 & 3.00 & $<0.001$ \\
\hline & No & 2234 & 99.33 & 226 & 97.00 & \\
\hline \multicolumn{7}{|l|}{ Injury } \\
\hline \multirow[t]{2}{*}{ Total } & Yes & 17 & 0.76 & 3 & 1.29 & 0.388 \\
\hline & No & 2232 & 99.24 & 230 & 98.71 & \\
\hline \multirow[t]{2}{*}{ Vascular } & Yes & 8 & 0.36 & 3 & 1.29 & 0.042 \\
\hline & No & 2241 & 99.64 & 230 & 98.71 & \\
\hline \multirow[t]{2}{*}{ Bowell } & Yes & 5 & 0.22 & 0 & 0.00 & 0.471 \\
\hline & No & 2244 & 99.78 & 233 & 100.0 & \\
\hline \multirow[t]{2}{*}{ Bladder } & Yes & 2 & 0.09 & 0 & 0.00 & 0.649 \\
\hline & No & 2247 & 99.91 & 233 & 100.0 & \\
\hline \multicolumn{7}{|l|}{ Postoperative complication } \\
\hline \multirow[t]{2}{*}{ Total } & Yes & 80 & 3.56 & 20 & 8.58 & $<0.001$ \\
\hline & No & 2169 & 96.44 & 213 & 91.42 & \\
\hline \multirow[t]{2}{*}{ Bleeding } & Yes & 29 & 1.29 & 6 & 2.58 & 0.113 \\
\hline & No & 2220 & 98.71 & 227 & 97.42 & \\
\hline \multirow[t]{2}{*}{ Seroma } & Yes & 51 & 2.27 & 14 & 6.01 & $<0.001$ \\
\hline & No & 2198 & 97.73 & 219 & 93.99 & \\
\hline \multirow[t]{2}{*}{ Infection } & Yes & 1 & 0.04 & 0 & 0.00 & 0.748 \\
\hline & No & 2248 & 99.96 & 233 & 100.0 & \\
\hline \multirow[t]{2}{*}{ Bowell injury } & Yes & 1 & 0.04 & 0 & 0.00 & 0.748 \\
\hline & No & 2248 & 99.96 & 233 & 100.0 & \\
\hline \multirow[t]{2}{*}{ Wound healing disorders } & Yes & 1 & 0.04 & 0 & 0.00 & 0.748 \\
\hline & No & 2248 & 99.96 & 233 & 100.0 & \\
\hline \multirow[t]{2}{*}{ Reoperations } & Yes & 27 & 1.20 & 6 & 2.58 & 0.081 \\
\hline & No & 2222 & 98.80 & 227 & 97.42 & \\
\hline \multirow[t]{2}{*}{ Recurrence on follow-up } & Yes & 28 & 1.24 & 8 & 3.43 & 0.008 \\
\hline & No & 2221 & 98.76 & 225 & 96.57 & \\
\hline \multirow[t]{2}{*}{ Pain in rest on follow-up } & Yes & 133 & 5.91 & 20 & 8.58 & 0.107 \\
\hline & No & 2116 & 94.09 & 213 & 91.42 & \\
\hline \multirow[t]{2}{*}{ Pain on exertion on follow-up } & Yes & 250 & 11.12 & 34 & 14.59 & 0.113 \\
\hline & No & 1999 & 88.88 & 199 & 85.41 & \\
\hline \multirow[t]{2}{*}{ Pain requiring treatment on follow-up } & Yes & 85 & 3.78 & 10 & 4.29 & 0.698 \\
\hline & No & 2164 & 96.22 & 223 & 95.71 & \\
\hline
\end{tabular}

The multivariable analysis results for pain at rest are presented in Table 19 (model matching: $p<0.001$ ). Here, the hernia defect size proved to be the strongest influence factor $(p=0.006)$. A larger recurrent hernia (II vs I: OR 0.521 [0.346; 0.786]; III vs I: OR 0.560 [0.352; 0.892]) reduced the risk of pain at rest.

Likewise, repair as per the guidelines $(p=0.016)$ and BMI ( $p=0.019)$ had a significant influence on pain at rest.
Repair as per the guidelines (OR 1.508 [1.079; 2.107]) as well as a five-point higher BMI (five-point OR 1.295 $[1.043 ; 1.609])$ increased the risk of pain at rest.

Another descriptive analysis revealed that the increased risk of pain at rest was attributed primarily to the smallsized $(<1.5 \mathrm{~cm})$ and medium-sized $(1.5-3 \mathrm{~cm})$ hernias (Table 20). 
Table 13 Multivariable analysis of postoperative complications in patients with laparo-endoscopic unilateral recurrent inguinal hernia repair

Table 14 Open unilateral recurrent inguinal hernia repairs on compliance versus noncompliance with the guidelines

\begin{tabular}{llllll}
\hline Parameter & $p$ value & Category & OR estimate & 95\% CI \\
\hline Guidelines & 0.001 & Yes versus no & 0.419 & 0.248 & 0.708 \\
Defect size & 0.018 & II $(1.5-3 \mathrm{~cm})$ versus I $(<1.5 \mathrm{~cm})$ & 1.256 & 0.656 & 2.404 \\
& & III $(>3 \mathrm{~cm})$ versus I $(<1.5 \mathrm{~cm})$ & 2.329 & 1.135 & 4.779 \\
Age (10-year OR) & 0.089 & & 1.152 & 0.979 & 1.357 \\
EHS-classification medial & 0.115 & Yes versus no & 0.572 & 0.285 & 1.146 \\
Risk factor & 0.269 & Yes versus no & 1.293 & 0.820 & 2.038 \\
BMI (five-point OR) & 0.420 & & 0.876 & 0.634 & 1.210 \\
EHS-classification femoral & 0.429 & Yes versus no & 1.485 & 0.558 & 3.953 \\
EHS-classification lateral & 0.532 & Yes versus no & 0.797 & 0.392 & 1.621 \\
EHS-classification scrotal & 0.612 & Yes versus no & 1.378 & 0.399 & 4.758 \\
ASA score & 0.657 & II versus I & 0.849 & 0.484 & 1.489 \\
& & III/IV versus I & 1.056 & 0.512 & 2.179 \\
\hline
\end{tabular}

\begin{tabular}{|c|c|c|c|c|c|c|c|c|}
\hline & \multicolumn{6}{|c|}{ Previous operations } & \multirow{2}{*}{\multicolumn{2}{|c|}{ Total }} \\
\hline & \multicolumn{2}{|l|}{ Suture } & \multicolumn{2}{|c|}{ Open mesh } & \multicolumn{2}{|c|}{ Endoscopic mesh } & & \\
\hline & $N$ & ColPctN & $N$ & ColPctN & $N$ & ColPctN & $N$ & ColPctN \\
\hline \multicolumn{9}{|c|}{ Guidelines } \\
\hline No & 1011 & 100.0 & 412 & 100.0 & - & - & 1423 & 61.3 \\
\hline Yes & - & - & - & - & 897 & 100.0 & 897 & 38.7 \\
\hline Total & 1011 & 100.0 & 412 & 100.0 & 897 & 100.0 & 2320 & 100.0 \\
\hline
\end{tabular}

Table 15 Age and BMI of patients with open unilateral recurrent inguinal hernia repair on compliance versus non-compliance with the guidelines

\begin{tabular}{lllll}
\hline & & \multirow{2}{l}{ Guidelines } & \multirow{2}{*}{$p$} \\
\cline { 3 - 4 } & & Yes & No & \\
\hline Age (years) & Mean \pm STD & $59.3 \pm 13.5$ & $62.5 \pm 16.2$ & $<0.001$ \\
BMI & Mean \pm STD & $26.3 \pm 3.6$ & $25.8 \pm 3.4$ & $<0.001$ \\
\hline
\end{tabular}

The multivariable analysis results for pain on exertion are illustrated in Table 21 (model matching: $p<0.001$ ). These were significantly influenced by the hernia defect size $(p=0.002)$, repair as per the guidelines $(p=0.010)$, BMI $(p=0.023)$, age $(p=0.027)$ and scrotal EHS classification $(p=0.036)$. A higher age (10-year OR 0.897 [0.814; 0.988]), larger hernias (II vs I: OR 0.654 [0.475; $0.901]$; III vs I: OR 0.517 [0.335; 0.754]) as well as scrotal EHS classification (OR 0.211 [0.049; 0.900]) reduced the risk of pain on exertion. Conversely, there was a higher risk of pain for repair as per the guidelines (OR 1.401 [1.084; 1.810]) and for a five-point larger BMI (five-point OR $1.224[1.029 ; 1.456])$. Likewise, for pain on exertion the risk was attributable, in particular, to small-sized $(<1.5 \mathrm{~cm})$ and medium-sized $(1.5-3 \mathrm{~cm})$ recurrent hernias (Table 22).

\section{Discussion}

1. The present analysis of data from the Herniamed Registry [29] first investigated to what extent participants in the Herniamed Hernia Registry [29] complied with the recommendations set out in the guidelines of the European Hernia Society (EHS). This revealed that laparo-endoscopic recurrent repair was used in $61.6 \%$ of cases following previous open suture repair and in $28.9 \%$ cases following open mesh repair as well as in $9.4 \%$ of cases following previous laparo-endoscopic operations. Hence, more than $90 \%$ of laparo-endoscopic recurrent repair procedures were performed in accordance with the EHS guidelines. Only $9.4 \%$ did not comply with the guidelines.

Matters were different for open recurrent repair. Only $38.5 \%$ of open recurrent repair operations were conducted following primary laparo-endoscopic repair. $43.4 \%$ of open recurrent repair procedures were performed following previous open suture repair and $17.7 \%$ following previous open mesh repair. As such, more than $60 \%$ of open recurrent operations did not comply with the recommendations of the guidelines. Already Richards et al. [13] and Richards and Earnshaw [28] pointed out that surgeons using predominantly open hernia surgery techniques also use predominantly open surgery for recurrent repair. It appears that the guidelines, which were first published in 
Table 16 Demographic and surgery-related parameters and risk factors for patients with open unilateral recurrent inguinal hernia repair on compliance versus noncompliance with the guidelines

\begin{tabular}{|c|c|c|c|c|c|c|}
\hline & & \multicolumn{4}{|c|}{ Guidelines } & \multirow[t]{3}{*}{$p$} \\
\hline & & \multicolumn{2}{|l|}{ Yes } & \multicolumn{2}{|l|}{ No } & \\
\hline & & $n$ & $\%$ & $n$ & $\%$ & \\
\hline \multirow[t]{3}{*}{ ASA score } & I & 258 & 28.45 & 368 & 25.86 & \multirow[t]{3}{*}{$<0.001$} \\
\hline & II & 509 & 56.12 & 708 & 49.75 & \\
\hline & III/IV & 140 & 15.44 & 347 & 24.39 & \\
\hline \multirow[t]{3}{*}{ Defect size } & $\mathrm{I}(<1.5 \mathrm{~cm})$ & 154 & 16.98 & 240 & 16.87 & \multirow[t]{3}{*}{0.028} \\
\hline & II $(1.5-3 \mathrm{~cm})$ & 498 & 54.91 & 711 & 49.96 & \\
\hline & III $(>3 \mathrm{~cm})$ & 255 & 28.11 & 472 & 33.17 & \\
\hline \multicolumn{7}{|l|}{ Risk factor } \\
\hline \multirow[t]{2}{*}{ Total } & Yes & 277 & 30.54 & 559 & 39.28 & \multirow[t]{2}{*}{$<0.001$} \\
\hline & No & 630 & 69.46 & 864 & 60.72 & \\
\hline \multirow[t]{2}{*}{ COPD } & Yes & 67 & 7.39 & 149 & 10.47 & \multirow[t]{2}{*}{0.012} \\
\hline & No & 840 & 92.61 & 1274 & 89.53 & \\
\hline \multirow[t]{2}{*}{ Diabetes } & Yes & 51 & 5.62 & 114 & 8.01 & \multirow[t]{2}{*}{0.028} \\
\hline & No & 856 & 94.38 & 1309 & 91.99 & \\
\hline \multirow[t]{2}{*}{ Aortic aneurism } & Yes & 4 & 0.44 & 11 & 0.77 & \multirow[t]{2}{*}{0.329} \\
\hline & No & 903 & 99.56 & 1412 & 99.23 & \\
\hline \multirow[t]{2}{*}{ Immunosuppression } & Yes & 10 & 1.10 & 23 & 1.62 & \multirow[t]{2}{*}{0.306} \\
\hline & No & 897 & 98.90 & 1400 & 98.38 & \\
\hline \multirow[t]{2}{*}{ Corticoid } & Yes & 8 & 0.88 & 29 & 2.04 & \multirow[t]{2}{*}{0.030} \\
\hline & No & 899 & 99.12 & 1394 & 97.96 & \\
\hline \multirow[t]{2}{*}{ Smoking } & Yes & 111 & 12.24 & 203 & 14.27 & \multirow[t]{2}{*}{0.162} \\
\hline & No & 796 & 87.76 & 1220 & 85.73 & \\
\hline \multirow[t]{2}{*}{ Coagulopathy } & Yes & 9 & 0.99 & 40 & 2.81 & \multirow[t]{2}{*}{0.003} \\
\hline & No & 898 & 99.01 & 1383 & 97.19 & \\
\hline \multirow[t]{2}{*}{ Antiplatelet medication } & Yes & 79 & 8.71 & 186 & 13.07 & \multirow[t]{2}{*}{0.001} \\
\hline & No & 828 & 91.29 & 1237 & 86.93 & \\
\hline Anticoagulation therapy & Yes & 25 & 2.76 & 50 & 3.51 & 0.313 \\
\hline & No & 882 & 97.24 & 1373 & 96.49 & \\
\hline EHS-classification medial & Yes & 523 & 57.66 & 795 & 55.87 & 0.394 \\
\hline & No & 384 & 42.34 & 628 & 44.13 & \\
\hline EHS-classification lateral & Yes & 460 & 50.72 & 800 & 56.22 & 0.009 \\
\hline & No & 447 & 49.28 & 623 & 43.78 & \\
\hline EHS-classification femoral & Yes & 15 & 1.65 & 32 & 2.25 & 0.319 \\
\hline & No & 892 & 98.35 & 1391 & 97.75 & \\
\hline EHS-classification scrotal & Yes & 12 & 1.32 & 63 & 4.43 & $<0.001$ \\
\hline & No & 895 & 98.68 & 1360 & 95.57 & \\
\hline
\end{tabular}

2009 [23], have not changed that scenario. Further highquality studies are needed to demonstrate that repair as per the guidelines really does achieve a better outcome for patients. Only when convincing evidence based on highquality trials is available can greater acceptance of the guidelines be expected. Since to date no such studies have been carried out, it is no surprise that surgeons have called upon their own expertise when deciding on the surgical technique used to treat patients with recurrent inguinal hernia. Guidelines always only reflect the current state of knowledge gained from the studies reported in the scientific literature. If new published data are added, the recommendations may also change. Mere deviation from a guideline is unlikely to be considered as malpractice in litigation, unless the practice concerned is so well established that no responsible surgeon would fail to adhere to it [31].

2. To date, no study has compared the outcomes of recurrent inguinal hernia repair carried out in compliance with the guidelines. Therefore, the present analysis of Herniamed data [29] compared laparo-endoscopic with open recurrent repair performed as per the guidelines. No 
Table 17 Intra- and postoperative complications, complication-related reoperations and 1-year followup results of patients with open unilateral recurrent inguinal hernia repair on compliance versus non-compliance with the guidelines

\begin{tabular}{|c|c|c|c|c|c|c|}
\hline & & \multicolumn{2}{|l|}{ Yes } & \multicolumn{2}{|l|}{ No } & \multirow[t]{2}{*}{$p$} \\
\hline & & $n$ & $\%$ & $n$ & $\%$ & \\
\hline \multicolumn{7}{|l|}{ Intraoperative complication } \\
\hline \multirow[t]{2}{*}{ Total } & Yes & 14 & 1.54 & 23 & 1.62 & 0.891 \\
\hline & No & 893 & 98.46 & 1400 & 98.38 & \\
\hline \multirow[t]{2}{*}{ Bleeding } & Yes & 3 & 0.33 & 12 & 0.84 & 0.131 \\
\hline & No & 904 & 99.67 & 1411 & 99.16 & \\
\hline \multicolumn{7}{|l|}{ Injury } \\
\hline \multirow[t]{2}{*}{ Total } & Yes & 12 & 1.32 & 14 & 0.98 & 0.447 \\
\hline & No & 895 & 98.68 & 1409 & 99.02 & \\
\hline \multirow[t]{2}{*}{ Vascular } & Yes & 0 & 0.00 & 3 & 0.21 & 0.166 \\
\hline & No & 907 & 100.0 & 1420 & 99.79 & \\
\hline \multirow[t]{2}{*}{ Bowell } & Yes & 0 & 0.00 & 4 & 0.28 & 0.110 \\
\hline & No & 907 & 100.0 & 1419 & 99.72 & \\
\hline \multirow[t]{2}{*}{ Bladder } & Yes & 1 & 0.11 & 1 & 0.07 & 0.748 \\
\hline & No & 906 & 99.89 & 1422 & 99.93 & \\
\hline \multirow[t]{2}{*}{ Nerve } & Yes & 9 & 0.99 & 1 & 0.07 & $<0.001$ \\
\hline & No & 898 & 99.01 & 1422 & 99.93 & \\
\hline \multicolumn{7}{|l|}{ Postoperative complication } \\
\hline \multirow[t]{2}{*}{ Total } & Yes & 33 & 3.64 & 82 & 5.76 & 0.021 \\
\hline & No & 874 & 96.36 & 1341 & 94.24 & \\
\hline \multirow[t]{2}{*}{ Bleeding } & Yes & 17 & 1.87 & 45 & 3.16 & 0.060 \\
\hline & No & 890 & 98.13 & 1378 & 96.84 & \\
\hline \multirow[t]{2}{*}{ Seroma } & Yes & 14 & 1.54 & 30 & 2.11 & 0.329 \\
\hline & No & 893 & 98.46 & 1393 & 97.89 & \\
\hline \multirow[t]{2}{*}{ Infection } & Yes & 0 & 0.00 & 3 & 0.21 & 0.166 \\
\hline & No & 907 & 100.0 & 1420 & 99.79 & \\
\hline \multirow[t]{2}{*}{ Wound healing disorders } & Yes & 4 & 0.44 & 7 & 0.49 & 0.861 \\
\hline & No & 903 & 99.56 & 1416 & 99.51 & \\
\hline \multirow[t]{2}{*}{ Reoperation } & Yes & 9 & 0.99 & 30 & 2.11 & 0.041 \\
\hline & No & 898 & 99.01 & 1393 & 97.89 & \\
\hline \multirow[t]{2}{*}{ Recurrence on follow-up } & Yes & 10 & 1.10 & 37 & 2.60 & 0.012 \\
\hline & No & 897 & 98.90 & 1386 & 97.40 & \\
\hline \multirow[t]{2}{*}{ Pain in rest on follow-up } & Yes & 78 & 8.60 & 77 & 5.41 & 0.003 \\
\hline & No & 829 & 91.40 & 1346 & 94.59 & \\
\hline \multirow[t]{2}{*}{ Pain on exertion on follow-up } & Yes & 136 & 14.99 & 145 & 10.19 & $<0.001$ \\
\hline & No & 771 & 85.01 & 1278 & 89.81 & \\
\hline \multirow[t]{2}{*}{ Pain requiring treatment on follow-up } & Yes & 40 & 4.41 & 50 & 3.51 & 0.274 \\
\hline & No & 867 & 95.59 & 1373 & 96.49 & \\
\hline
\end{tabular}

significant difference was identified between laparo-endoscopic and open techniques performed as per the guidelines in terms of the overall intraoperative complication rate, postoperative complication rate, complication-related reoperation rate, recurrence rate and rate of chronic pain requiring treatment. However, with regard to the intraoperative complications open recurrent repair was associated with significantly more nerve injuries as well as more pain at rest and pain on exertion on 1-year follow-up.
Multivariable analysis confirmed that laparo-endoscopic repair had a significant impact on pain at rest and pain on exertion, and was associated with a lower pain rate compared with open recurrent repair. Even on compliance with the guidelines, a significantly higher rate of pain at rest and pain on exertion must be expected when open repair is used following previous laparo-endoscopic operations compared with laparo-endoscopic repair after previous open repair. Therefore, such recurrent repair operations should be 
Table 18 Multivariable analysis of postoperative complications in patients with open unilateral recurrent inguinal hernia repair
Table 19 Multivariable analysis of pain at rest in patients with open unilateral recurrent inguinal hernia repair

\begin{tabular}{llllll}
\hline Parameter & $p$ value & Category & OR estimate & \multicolumn{2}{l}{ 95\% CI } \\
\hline Age (10-year OR) & 0.003 & & 1.275 & 1.085 & 1.498 \\
Risk factor & 0.118 & Yes versus no & 1.390 & 0.919 & 2.102 \\
Guidelines & 0.155 & Yes versus no & 0.734 & 0.479 & 1.124 \\
EHS-classification lateral & 0.165 & Yes versus no & 0.654 & 0.359 & 1.191 \\
Defect size & 0.181 & II $(1.5-3 \mathrm{~cm})$ versus I $(<1.5 \mathrm{~cm})$ & 0.718 & 0.420 & 1.225 \\
& & III $(>3 \mathrm{~cm})$ versus I $(<1.5 \mathrm{~cm})$ & 1.053 & 0.600 & 1.848 \\
EHS-classification medial & 0.225 & Yes versus no & 0.685 & 0.372 & 1.262 \\
BMI (five-point OR) & 0.392 & & 0.880 & 0.656 & 1.180 \\
ASA score & 0.434 & II versus I & 0.742 & 0.439 & 1.256 \\
& & III/IV versus I & 0.913 & 0.470 & 1.775 \\
EHS-classification femoral & 0.935 & Yes versus no & 0.950 & 0.276 & 3.275 \\
EHS-classification scrotal & 0.975 & Yes versus no & 0.985 & 0.371 & 2.612 \\
\hline
\end{tabular}

\begin{tabular}{llllll}
\hline Parameter & $p$ value & Category & OR estimate & \multicolumn{2}{l}{$95 \%$ CI } \\
\hline Defect size & 0.006 & II $(1.5-3 \mathrm{~cm})$ versus I $(<1.5 \mathrm{~cm})$ & 0.521 & 0.346 & 0.786 \\
& & III $(>3 \mathrm{~cm})$ versus I $(<1.5 \mathrm{~cm})$ & 0.560 & 0.352 & 0.892 \\
Guidelines & 0.016 & Yes versus no & 1.508 & 1.079 & 2.107 \\
BMI (five-point OR) & 0.019 & & 1.295 & 1.043 & 1.609 \\
Age (10-year OR) & 0.110 & & 0.902 & 0.795 & 1.023 \\
EHS-classification femoral & 0.164 & Yes versus no & 0.238 & 0.032 & 1.798 \\
EHS-classification lateral & 0.243 & Yes versus no & 0.716 & 0.409 & 1.254 \\
EHS-classification medial & 0.352 & Yes versus no & 0.761 & 0.428 & 1.353 \\
ASA score & 0.490 & II versus I & 0.829 & 0.556 & 1.236 \\
& & III/IV versus I & 0.697 & 0.375 & 1.295 \\
Risk factor & 0.528 & Yes versus no & 1.126 & 0.779 & 1.628 \\
EHS-classification scrotal & 0.756 & Yes versus no & 0.839 & 0.276 & 2.545 \\
\hline
\end{tabular}

Table 20 Correlation of the defect size, compliance versus non-compliance with the guidelines and pain in rest on follow-up in patients with open unilateral recurrent inguinal hernia repair

\begin{tabular}{|c|c|c|c|c|c|c|c|c|c|}
\hline & & \multicolumn{6}{|c|}{ Defect size } & \multirow{2}{*}{\multicolumn{2}{|c|}{ All }} \\
\hline & & \multicolumn{2}{|c|}{$\mathrm{I}(<1.5 \mathrm{~cm})$} & \multicolumn{2}{|c|}{ II $(1.5-3 \mathrm{~cm})$} & \multicolumn{2}{|c|}{ III $(>3 \mathrm{~cm})$} & & \\
\hline & & $N$ & $\%$ & $N$ & $\%$ & $N$ & $\%$ & $N$ & $\%$ \\
\hline Guidelines & Pain in rest on follow-up & & & & & & & & \\
\hline \multirow[t]{2}{*}{ No } & No & 217 & 90.4 & 685 & 96.3 & 444 & 94.1 & 1346 & 94.6 \\
\hline & Yes & 23 & 9.6 & 26 & 3.7 & 28 & 5.9 & 77 & 5.4 \\
\hline \multirow[t]{2}{*}{ Yes } & No & 135 & 87.7 & 455 & 91.4 & 239 & 93.7 & 829 & 91.4 \\
\hline & Yes & 19 & 12.3 & 43 & 8.6 & 16 & 6.3 & 78 & 8.6 \\
\hline
\end{tabular}

performed by surgeons who are highly experienced in the respective technique. Therefore, despite observance of the guidelines, higher rates of pain at rest and pain on exertion must be expected on using open recurrent repair following primary laparo-endoscopic repair than when using laparoendoscopic recurrent repair following primary open repair.

3. In particular, since a large number of open $(61.1 \%)$ and also a smaller number of laparo-endoscopic (9.4\%) recurrent repair procedures were not performed in accordance with the recommendations of the guidelines, the question arises as to how the outcomes compare with the respective repair procedures carried out in compliance with the guidelines.

If recurrent repair is conducted as per the guidelines, laparo-endoscopic repair is associated with fewer intraoperative and postoperative complications and with a lower re-recurrence rate. No difference was found for the pain rates. Multivariable analysis demonstrated especially for the postoperative complications the impact of repair as per the guidelines. 
Table 21 Multivariable analysis of pain on exertion in patients with open unilateral recurrent inguinal hernia repair

Table 22 Correlation of the defect size, compliance versus non-compliance with the guidelines and pain on exertion on follow-up in patients with open unilateral recurrent inguinal hernia repair

\begin{tabular}{llllll}
\hline Parameter & $p$ value & Category & OR estimate & \multicolumn{2}{l}{ 95\% CI } \\
\hline Defect size & 0.002 & II $(1.5-3 \mathrm{~cm})$ versus I $(<1.5 \mathrm{~cm})$ & 0.654 & 0.475 & 0.901 \\
& & III $(>3 \mathrm{~cm})$ versus I $(<1.5 \mathrm{~cm})$ & 0.517 & 0.355 & 0.754 \\
Guidelines & 0.010 & Yes versus no & 1.401 & 1.084 & 1.810 \\
BMI (five-point OR) & 0.023 & & 1.224 & 1.029 & 1.456 \\
Age (10-year OR) & 0.027 & & 0.897 & 0.814 & 0.988 \\
EHS-classification scrotal & 0.036 & Yes versus no & 0.211 & 0.049 & 0.900 \\
EHS-classification lateral & 0.054 & Yes versus no & 0.653 & 0.423 & 1.007 \\
Risk factor & 0.241 & Yes versus no & 1.182 & 0.894 & 1.563 \\
EHS-classification femoral & 0.247 & Yes versus no & 0.531 & 0.182 & 1.551 \\
EHS-classification medial & 0.292 & Yes versus no & 0.787 & 0.504 & 1.229 \\
ASA score & 0.715 & II versus I & 1.054 & 0.769 & 1.446 \\
& & III/IV versus I & 0.905 & 0.563 & 1.453 \\
\hline
\end{tabular}

\begin{tabular}{|c|c|c|c|c|c|c|c|c|c|}
\hline & & \multicolumn{6}{|c|}{ Defect size } & \multirow{2}{*}{\multicolumn{2}{|c|}{ All }} \\
\hline & & \multicolumn{2}{|c|}{$\mathrm{I}(<1.5 \mathrm{~cm})$} & \multicolumn{2}{|c|}{ II $(1.5-3 \mathrm{~cm})$} & \multicolumn{2}{|c|}{ III $(>3 \mathrm{~cm})$} & & \\
\hline & & $N$ & $\%$ & $N$ & $\%$ & $N$ & $\%$ & $N$ & $\%$ \\
\hline \multirow{3}{*}{$\begin{array}{l}\text { Guidelines } \\
\text { No }\end{array}$} & Pain on exertion on follow-up & & & & & & & & \\
\hline & No & 204 & 85.0 & 644 & 90.6 & 430 & 91.1 & 1278 & 89.8 \\
\hline & Yes & 36 & 15.0 & 67 & 9.4 & 42 & 8.9 & 145 & 10.2 \\
\hline \multirow[t]{2}{*}{ Yes } & No & 121 & 78.6 & 421 & 84.5 & 229 & 89.8 & 771 & 85.0 \\
\hline & Yes & 33 & 21.4 & 77 & 15.5 & 26 & 10.2 & 136 & 15.0 \\
\hline
\end{tabular}

Comparison of open recurrent repair conducted on compliance versus non-compliance with the guidelines revealed fewer postoperative complications and complication-related reoperation rates as well as a lower re-recurrence rate following repair as per the guidelines. On the other hand, the risk of pain at rest and on exertion was higher on compliance with the guidelines. Multivariable analysis revealed that the postoperative complications were only affected by age but not by the use of a repair procedure in accordance with the guidelines. Matters were different for pain at rest and pain on exertion. For the latter, multivariable analysis confirmed that repair as per the guidelines exerted a significantly negative effect on onset of pain at rest and pain on exertion. However, multivariable analysis as well as an additional analysis demonstrated that a small defect size had the greatest impact on the risk of pain at rest and pain on exertion. Likewise, a higher BMI negatively impacted the risk of pain at rest and pain on exertion. Although recommended in the guidelines, patients with a small defect size and a higher BMI have a higher risk of pain at rest and exertion following open repair of a recurrence after a previous laparo-endoscopic inguinal hernia repair. Therefore, sufficient diagnostic work-up of a small recurrence as cause of groin pain is mandatory.
In summary, it can be stated that in the Herniamed Registry (1) $90 \%$ of the laparo-endoscopic and only $40 \%$ of open recurrent inguinal hernia repair operations are carried out in accordance with the EHS guidelines; (2) comparison of laparo-endoscopic with open recurrent repair conducted in accordance with the guidelines demonstrated that open recurrent repair as per the guidelines was associated with a higher risk of pain at rest and pain on exertion on 1-year follow-up; and (3) finally, comparison of recurrent repair procedures on compliance versus non-compliance with the guidelines showed that both laparo-endoscopic and open repair operations that did not comply with the guidelines presented a higher risk of perioperative complications and re-recurrences. As such, the recommendations set out in the EHS guidelines should be implemented, but considering the specific circumstances of a given patient.

Acknowledgements Ferdinand Köckerling has got grants to fund the Herniamed Registry from Johnson and Johnson, Norderstedt, Karl Storz, Tuttlingen, pfm medical, Cologne, Dahlhausen, Cologne, B Braun, Tuttlingen, MenkeMed, Munich, Bard, Karlsruhe and Resorba Medical GmbH, Nuremberg.

Herniamed Study Group Scientific Board Köckerling, Ferdinand (Chairman) (Berlin); Bittner, Reinhard (Rottenburg); Fortelny, René (Wien); Jacob, Dietmar (Berlin); Koch, Andreas (Cottbus); Kraft, Barbara (Stuttgart); Kuthe, Andreas (Hannover); Lippert, Hans 
(Magdeburg): Lorenz, Ralph (Berlin); Mayer, Franz (Salzburg); Moesta, Kurt Thomas (Hannover); Niebuhr, Henning (Hamburg); Peiper, Christian (Hamm); Pross, Matthias (Berlin); Reinpold, Wolfgang (Hamburg); Simon, Thomas (Weinheim); Stechemesser, Bernd (Köln); Unger, Solveig (Chemnitz). Participants Ahmetov, Azat (Saint-Petersburg); Alapatt, Terence Francis (Frankfurt/Main); Albayrak, Nurettin (Herne); Amann, Stefan (Neuendettelsau); Anders, Stefan (Berlin); Anderson, Jürina (Würzburg); Antoine, Dirk (Leverkusen); Arndt, Anatoli (Elmshorn); Asperger, Walter (Halle); Avram, Iulian (Saarbrücken); Baikoglu-Endres, Corc (Weißenburg i. Bay.); Bandowsky, Boris (Damme); Barkus; Jörg (Velbert); Becker, Matthias (Freital); Behrend, Matthias (Deggendorf); Beuleke, Andrea (Burgwedel); Berger, Dieter (Baden-Baden); Birk, Dieter (Bietigheim-Bissingen); Bittner, Reinhard (Rottenburg); Blaha, Pavel (Zwiesel); Blumberg, Claus (Lübeck); Böckmann, Ulrich (Papenburg); Böhle, Arnd Steffen (Bremen); Bolle, Ludger (Berlin); Borchert, Erika (Grevenbroich); Born, Henry (Leipzig); Brabender, Jan (Köln); Breitenbuch von, Philipp (Radebeul); Brož, Miroslav (Ebersbach); Brütting, Alfred (Erlangen); Buchert, Annette (Mallersdorf-Pfaffenberg); Budzier, Eckhard (Meldorf); Burchett, Bert (Waren); Burghardt, Jens (Rüdersdorf); Cejnar, StephanAlexander (München); Chirikov, Ruslan (Dorsten); Claußnitzer, Christian (Ulm); Comman, Andreas (Bogen); Crescenti, Fabio (Verden/Aller); Daniels, Thies (Hamburg); Dapunt, Emanuela (Bruneck); Decker, Georg (Berlin); Demmel, Michael (Arnsberg); Descloux, Alexandre (Baden); Deusch, Klaus-Peter (Wiesbaden); Dick, Marcus (Neumünster); Dieterich, Klaus (Ditzingen); Dietz, Harald (Landshut); Dittmann, Michael (Northeim); Drummer, Bernhard (Forchheim); Eckermann, Oliver (Luckenwalde); Eckhoff, Jörn/Hamburg); Ehmann, Frank (Grünstadt); Eisenkrein, Alexander (Düren); Elger, Karlheinz (Germersheim); Engelhardt, Thomas (Erfurt); Erichsen, Axel (Friedrichshafen); Eucker, Dietmar (Bruderholz); Fackeldey, Volker (Kitzingen); Farke, Stefan (Delmenhorst); Faust, Hendrik (Emden); Federmann, Georg (Seehausen); Feichter, Albert (Wien); Fiedler, Michael (Eisenberg); Fikatas, Panagiotis (Berlin); Firl, Michaela (Perleberg); Fischer, Ines (Wiener Neustadt); Fleischer, Sabine (Dinslaken); Fortelny, René H. (Wien); Franczak, Andreas (Wien); Franke, Claus (Düsseldorf); Frankenberg von, Moritz (Salem); Frehner, Wolfgang (Ottobeuren); Friedhoff, Klaus (Andernach); Friedrich, Jürgen (Essen); Frings, Wolfram (Bonn); Fritsche, Ralf (Darmstadt); Frommhold, Klaus (Coesfeld); Frunder, Albrecht (Tübingen); Fuhrer, Günther (Reutlingen); Gassler, Harald (Villach); Gawad, Karim A. Frankfurt/Main); Gehrig, Tobias (Sinsheim); Gerdes, Martin (Ostercappeln); Germanov, German (Halberstadt; Gilg, Kai-Uwe (Hartmannsdorf); Glaubitz, Martin (Neumünster); Glauner-Goldschmidt, Kerstin (Werne); Glutig, Holger (Meissen); Gmeiner, Dietmar (Bad Dürrnberg); Göring, Herbert (München); Grebe, Werner (Rheda-Wiedenbrück); Grothe, Dirk (Melle); Gürtler, Thomas (Zürich); Hache, Helmer (Löbau); Hämmerle, Alexander (Bad Pyrmont); Haffner, Eugen (Hamm); Hain, Hans-Jürgen (GrossUmstadt); Hammans, Sebastian (Lingen); Hampe, Carsten (Garbsen); Hanke, Stefan (Halle); Harrer, Petra (Starnberg); Hartung, Peter (Werne); Heinzmann, Bernd (Magdeburg); Heise, Joachim Wilfried (Stolberg); Heitland, Tim (München); Helbling, Christian (Rapperswil); Hempen, Hans-Günther (Cloppenburg); Henneking, KlausWilhelm (Bayreuth); Hennes, Norbert (Duisburg); Hermes, Wolfgang (Weyhe); Herrgesell, Holger (Berlin); Herzing, Holger Höchstadt); Hessler, Christian (Bingen); Heuer, Matthias (Herten); Hildebrand, Christiaan (Langenfeld); Höferlin, Andreas (Mainz); Hoffmann, Henry (Basel); Hoffmann, Michael (Kassel); Hofmann, Eva M. (Frankfurt/Main); Hornung, Frederic (Wolfratshausen); Hügel, Omar (Hannover); Hüttemann, Martin (Oberhausen); Hunkeler, Rolf (Zürich); Imdahl, Andreas (Heidenheim); Isemer, Friedrich-Eckart (Wiesbaden); Jablonski, Herbert Gustav (Sögel); Jacob, Dietmar (Berlin); Jansen-Winkeln, Boris (Leipzig); Jantschulev, Methodi
(Waren); Jenert, Burghard (Lichtenstein); Jugenheimer, Michael (Herrenberg); Junger, Marc (München); Kaaden, Stephan (Neustadt am Rübenberge); Käs, Stephan (Weiden); Kahraman, Orhan (Hamburg); Kaiser, Christian (Westerstede); Kaiser, Gernot Maximilian (Kamp-Lintfort); Kaiser, Stefan (Kleinmachnow); Kapischke, Matthias (Hamburg); Karch, Matthias (Eichstätt); Kasparek, Michael S. (München); Keck, Heinrich (Wolfenbüttel); Keller, Hans W. (Bonn); Kienzle, Ulrich (Karlsruhe); Kipfmüller, Brigitte (Köthen); Kirsch, Ulrike (Oranienburg); Klammer, Frank (Ahlen); Klatt, Richard (Hagen); Klein, Karl-Hermann (Burbach); Kleist, Sven (Berlin); Klobusicky, Pavol (Bad Kissingen); Kneifel, Thomas (Datteln); Knoop, Michael (Frankfurt/Oder); Knotter, Bianca (Mannheim); Koch, Andreas (Cottbus); Koch, Andreas (Münster); Köckerling, Ferdinand (Berlin); Köhler, Gernot (Linz); König, Oliver (Buchholz); Kornblum, Hans (Tübingen); Krämer, Dirk (Bad Zwischenahn); Kraft, Barbara (Stuttgart); Kratsch, Barthel (Dierdorf/Selters); Kreissl, Peter (Ebersberg); Krones, Carsten Johannes (Aachen); Kronhardt, Heinrich (Neustadt am Rübenberge); Kruse, Christinan (Aschaffenburg); Kube, Rainer (Cottbus); Kühlberg, Thomas (Berlin); Kühn, Gert (Freiberg); Kuhn, Roger (Gifhorn); Kusch, Eduard (Gütersloh); Kuthe, Andreas (Hannover); Ladberg, Ralf (Bremen); Ladra, Jürgen (Düren); Lahr-Eigen, Rolf (Potsdam); Lainka, Martin (Wattenscheid); Lammers, Bernhard J. (Neuss); Lancee, Steffen (Alsfeld); Lange, Claas (Berlin); Langer, Claus (Göttingen); Laps, Rainer (Ehringshausen); Larusson, Hannes Jon (Pinneberg); Lauschke, Holger (Duisburg); Leher, Markus (Schärding); Leidl, Stefan (Waidhofen/ Ybbs); Lenz, Stefan (Berlin); Liedke, Marc Olaf (Heide); Lienert, Mark (Duisburg); Limberger, Andreas (Schrobenhausen); Limmer, Stefan (Würzburg); Locher, Martin (Kiel); Loghmanieh, Siawasch (Viersen); Lorenz, Ralph (Berlin); Luther, Stefan (Wipperfürth); Luyken, Walter (Sulzbach-Rosenberg); Mallmann, Bernhard (Krefeld); Manger, Regina (Schwabmünchen); Maurer, Stephan (Münster); May, Jens Peter (Schönebeck); Mayer, Franz (Salzburg); Mayer, Jens (Schwäbisch Gmünd); Mellert, Joachim (Höxter); Menzel, Ingo (Weimar); Meurer, Kirsten (Bochum); Meyer, Moritz (Ahaus); Mirow, Lutz (Kirchberg); Mittag-Bonsch, Martina (Crailsheim); Mittenzwey, Hans-Joachim (Berlin); Möbius, Ekkehard (Braunschweig); Mörder-Köttgen, Anja (Freiburg); Moesta, Kurt Thomas (Hannover); Moldenhauer, Ingolf (Braunschweig); Morkramer, Rolf (Xanten); Mosa, Tawfik (Merseburg); Müller, Hannes (Schlanders); Münzberg, Gregor (Berlin); Murr, Alfons (Vilshofen); Mussack, Thomas (St. Gallen); Nartschik, Peter (Quedlinburg); Nasifoglu, Bernd (Ehingen); Neumann, Jürgen (Haan); Neumeuer, Kai (Paderborn); Niebuhr, Henning (Hamburg); Nix, Carsten (Walsrode); Nölling, Anke (Burbach); Nostitz, Friedrich Zoltán (Mühlhausen); Obermaier, Straubing); Öz-Schmidt, Meryem (Hanau); Oldorf, Peter (Usingen); Olivieri, Manuel (Pforzheim); Passon, Marius (Freudenberg); Pawelzik, Marek (Hamburg); Pein, Tobias (Hameln); Peiper, Christian (Hamm); Peiper, Matthias (Essen); Peitgen, Klaus (Bottrop); Pertl, Alexander (Spittal/Drau); Philipp, Mark (Rostock); Pickart, Lutz (Bad Langensalza); Pizzera, Christian (Graz); Pöllath, Martin (Sulzbach-Rosenberg); Possin, Ulrich (Laatzen); Prenzel, Klaus (Bad Neuenahr-Ahrweiler); Pröve, Florian (Goslar); Pronnet, Thomas (Fürstenfeldbruck); Pross, Matthias (Berlin); Puff, Johannes (Dinkelsbühl); Rabl, Anton (Passau); Raggi, Matthias Claudius (Stuttgart); Rapp, Martin (Neunkirchen); Reck, Thomas (Püttlingen); Reinpold, Wolfgang (Hamburg); Reuter, Christoph (Quakenbrück); Richter, Jörg (Winnenden); Riemann, Kerstin (Alzenau-Wasserlos); Rodehorst, Anette (Otterndorf); Roehr, Thomas (Rödental); Rössler, Michael (Rüdesheim am Rhein); Roncossek, Bremerhaven); Rosniatowski, Rolland (Marburg); Roth Hartmut (Nürnberg); Sardoschau, Nihad (Saarbrücken); Sauer, Gottfried (Rüsselsheim); Sauer, Jörg (Arnsberg); Seekamp, Axel (Freiburg); Seelig, Matthias (Bad Soden); Seidel, Hanka (Eschweiler); Seiler, Christoph Michael (Warendorf); Seltmann, Cornelia (Hachenburg); Senkal, Metin (Witten); Shamiyeh, 
Andreas (Linz); Shang, Edward (München); Siemssen, Björn (Berlin); Sievers, Dörte (Hamburg); Silbernik, Daniel (Bonn); Simon, Thomas (Weinheim); Sinn, Daniel (Olpe); Sinner, Guy (Merzig); Sinning, Frank (Nürnberg); Smaxwil, Constatin Aurel (Stuttgart); Sörensen, Björn (Lauf an der Pegnitz); Syga, Günter (Bayreuth); Schabel, Volker (Kirchheim/Teck); Schadd, Peter (Euskirchen); Schassen von, Christian (Hamburg); Scheidbach, Hubert (Neustadt/ Saale); Schelp, Lothar (Wuppertal); Scherf, Alexander (Pforzheim); Scheuerlein, Hubert (Paderborn); Scheyer, Mathias (Bludenz); Schilling, André (Kamen); Schimmelpenning, Hendrik (Neustadt in Holstein); Schinkel, Svenja (Kempten); Schmid, Michael (Gera); Schmid, Thomas (Innsbruck); Schmidt, Ulf (Mechernich); Schmitz, Heiner (Jena); Schmitz, Ronald (Altenburg); Schöche, Jan (Borna); Schoenen, Detlef (Schwandorf); Schrittwieser, Rudolf/Bruck an der Mur); Schroll, Andreas (München); Schubert, Daniel (Saarbrücken); Schüder, Gerhard (Wertheim); Schultz, Christian (Bremen-Lesum); Schultz, Harald (Landstuhl); Schulze, Frank P. Mülheim an der Ruhr); Schulze, Thomas (Dessau-Roßlau); Schumacher, Franz-Josef (Oberhausen); Schwab, Robert (Koblenz); Schwandner, Thilo (Lich); Schwarz, Jochen Günter (Rottenburg); Schymatzek, Ulrich (Eitorf); Spangenberger, Wolfgang (Bergisch-Gladbach); Sperling, Peter (Montabaur); Staade, Katja (Düsseldorf); Staib, Ludger (Esslingen); Staikov, Plamen (Frankfurt am Main); Stamm, Ingrid (Heppenheim); Stark, Wolfgang (Roth); Stechemesser, Bernd (Köln); Steinhilper, Uz (München); Stengl, Wolfgang (Nürnberg); Stern, Oliver (Hamburg); Stöltzing, Oliver (Meißen); Stolte, Thomas (Mannheim); Stopinski, Jürgen (Schwalmstadt); Stratmann, Gerald (Goch); Stubbe, Hendrik (Güstrow/); Stülzebach, Carsten (Friedrichroda); Tepel, Jürgen (Osnabrück); Terzić, Alexander (Wildeshausen); Teske, Ulrich (Essen); Tichomirow, Alexej (Brühl); Tillenburg, Wolfgang (Marktheidenfeld); Timmermann, Wolfgang (Hagen); Tomov, Tsvetomir (Koblenz; Train, Stefan H. (Gronau); Trauzettel, Uwe (Plettenberg); Triechelt, Uwe (Langenhagen); Ulbricht, Wolfgang (Breitenbrunn); Ulcar, Heimo (Schwarzach im Pongau); Unger, Solveig (Chemnitz); Verweel, Rainer (Hürth); Vogel, Ulrike (Berlin); Voigt, Rigo (Altenburg); Voit, Gerhard (Fürth); Volkers, Hans-Uwe (Norden); Volmer, Ulla (Berlin); Vossough, Alexander (Neuss); Wallasch, Andreas (Menden); Wallner, Axel (Lüdinghausen); Warscher, Manfred (Lienz); Warwas, Markus (Bonn); Weber, Jörg (Köln); Weber, Uwe (Eggenfelden); Weihrauch, Thomas (Ilmenau); Weiß, Johannes (Schwetzingen); Weißenbach, Peter (Neunkirchen); Werner, Uwe (Lübbecke-Rahden); Wessel, Ina (Duisburg); Weyhe, Dirk (Oldenburg); Wieber, Isabell (Köln); Wiesmann, Aloys (Rheine); Wiesner, Ingo (Halle); Withöft, Detlef (Neutraubling); Woehe, Fritz (Sanderhausen); Wolf, Claudio (Neuwied); Wolkersdörfer, Toralf (Pößneck); Yaksan, Arif (Wermeskirchen); Yildirim, Can (Lilienthal); Yildirim, Selcuk (Berlin); Zarras, Konstantinos (Düsseldorf); Zeller, Johannes (Waldshut-Tiengen); Zhorzel, Sven (Agatharied); Zuz, Gerhard (Leipzig).

\section{Compliance with ethical standards}

Disclosures F. Köckerling, R. Bittner, A. Kuthe, B. Stechemesser, R. Lorenz, A. Koch, W. Reinpold, H. Niebuhr, M. Hukauf and C. SchugPass have no conflicts of interest or financial ties to disclose.

Open Access This article is distributed under the terms of the Creative Commons Attribution 4.0 International License (http://crea tivecommons.org/licenses/by/4.0/), which permits unrestricted use, distribution, and reproduction in any medium, provided you give appropriate credit to the original author(s) and the source, provide a link to the Creative Commons license, and indicate if changes were made.

\section{References}

1. Köckerling F, Jacob D, Wiegank W, Hukauf M, Schug-Pass C, Kuthe A, Bittner R (2016) Endoscopic repair of primary versus recurrent male unilateral inguinal hernias: are there differences in the outcome? Surg Endosc 30:1146-1155. doi:10.1007/s00464015-4318-3

2. Köckerling F, Koch A, Lorenz R, Reinpold W, Hukauf M, SchugPass C (2016) open repair of primary versus recurrent male unilateral inguinal hernias: perioperative complications and 1-year follow-up. World J Surg 40:813-825. doi:10.1007/s00268015-3325-9

3. Karthikesalingam A, Markar SR, Holt PJE, Praseedom RK (2010) Meta-analysis of randomized controlled trials comparing laparoscopic with open mesh recurrent inguinal hernia repair. $\mathrm{Br}$ J Surg 97:4-11. doi:10.1002/bjs.6902

4. Dedemadi G, Sgourakis G, Radtke A, Dounavis A, Gockel I, Fozas I, Karaliotas C, Anagnostou E (2010) Laparoscopic versus open mesh repair for recurrent inguinal hernia: a meta-analysis of outcomes. Am J Surg 200:291-297. doi:10.1016/j.amjsurg.2009.12.009

5. Yang J, Tong DN, Yao J, Chen W (2013) Laparoscopic or Lichtenstein repair for recurrent inguinal hernia: a meta-analysis of randomized controlled trials. ANZ J. Surg. 83:312-318. doi:10.1111/ans.12010

6. Pisanu A, Podda M, Saba A, Porceddu G, Uccheddu A (2015) Meta-analysis and review of prospective randomized trials comparing laparoscopic and Lichtenstein techniques in recurrent inguinal hernia repair. Hernia 19:355-366. doi:10.1007/s10029014-1281-1

7. Li J, Ji Z, Li Y (2014) Comparison of laparoscopic versus open procedure in the treatment of recurrent inguinal hernia: a metaanalysis of the results. Am J Surg 207:602-612. doi:10.1016/j. amjsurg.2013.05.008

8. Miserez M, Peeters E, Aufenacker T, Bouillot JL, Campanelli G, Conze J, Fortelny R, Heikkinen T, Jorgensen LN, Kukleta J, Morales-Conde S, Nordin P, Schumpelick V, Smedberg S, Smietanski M, Weber G, Simons MP (2014) Update with level 1 studies of the European Hernia Society guidelines on the treatment of inguinal hernia in adult patients. Hernia 18:151-163. doi:10.1007/s10029-014-1236-6

9. Kumar S, Nixon SL, MacIntyre IM (1999) Laparoscopic or Lichtenstein repair for recurrent inguinal hernia: one unit's experience. J R Coll Surg Edinb 44:301-302

10. Beets GL, Dirksen CD, Go PM, Geisler FE, Baeten CG, Kootstra G (1999) Open or laparoscopic preperitoneal mesh repair for recurrent inguinal hernia? A randomized controlled trial. Surg Endosc 13:323-327

11. Neumayer L, Giobbie-Hurder A, Jonasson O, Fitzgibbons R Jr, Dunlop D, Gibbs J, Reda D, Henderson W, Veterans Affairs Cooperative Studies Program 456 (2004) Open mesh versus laparoscopic mesh repair of inguinal hernia. $\mathrm{N}$ Engl J Med 350:1819-1827

12. Feliu X, Torres G, Viňas X, Martinez-Ródenas F, FernándezSallent E, Pie J (2004) Preperitoneal repair for recurrent inguinal hernia: laparoscopic and open approach. Hernia 8:113-116

13. Richards SK, Vipond MN, Earnshaw JJ (2004) Review of the management of recurrent inguinal hernia. Hernia 8:144-148

14. Rahr HB, Bendix J, Ahlburg P, Gjedsted J, Funch-Jensen P, Tønnesen E (2006) Coagulation, inflammatory, and stress responses in a randomized comparison of open and laparoscopic recurrent inguinal hernia repair. Surg Endosc 20:468-472

15. Dedemadi G, Sgourakis G, Karaliotas C, Christofides T, Kouraklis G, Karaliotas C (2006) Comparison of laparoscopic 
and open tension-free recurrent inguinal hernia repairs: a prospective randomized study. Surg Endosc 20:1099-1104

16. Alani A, Duffy F, O'Dwyer PJ (2006) Laparoscopic or open preperitoneal repair in the management of recurrent groin hernias. Hernia 10:156-158

17. Eklund A, Rudberg C, Leijonmarck CE, Rasmussen I, Spangen L, Wickbom G, Wingren U, Montgomery A (2007) Recurrent inguinal hernia: randomized multicenter trial comparing laparoscopic and Lichtenstein repair. Surg Endosc 21:634-640

18. Kouhia ST, Huttunen R, Silvasti SO, Heiskanen JT, Ahtola H, Uotila-Nieminen M, Kiviniemi VV, Hakala T (2009) Lichtenstein hernioplasty versus totally extraperitoneal laparoscopic hernioplasty in treatment of recurrent inguinal hernia: a prospective randomized trial. Ann Surg 249:384-387. doi:10. 1097/SLA.0b013e318196d0b0

19. Shah NR, Mikami DJ, Cook C, Manilchuk A, Hodges C, Memark VR, Volckmann ET, Hall CR, Steinberg S, Needleman B, Hazey JW, Melvin WS, Narula VK (2011) A comparison of outcomes between open and laparoscopic surgical repair of recurrent inguinal hernias. Surg Endosc 25:2330-2337. doi:10.1007/ 300464-010-1564-2

20. Demetrashvili Z, Qerqadze V, Kamkamidze G, Topchishvili G, Lagvilava L, Chartholani T, Archvadze V (2011) Comparison of Lichtenstein and laparoscopic transabdominal preperitoneal repair of recurrent inguinal hernias. Int Surg 96:233-238

21. Saber A, Hokkam EN, Ellabban GM (2015) Laparoscopic transabdominal preperitoneal approach for recurrent inguinal hernia: a randomized trial. J. Minim. Access. Surg. 11:123-128

22. Sevonius D, Montgomery A, Smedberg S, Sandblom G (2016) Chronic groin pain, discomfort and physical disability after recurrent groin hernia repair: impact of anterior and posterior mesh repair. Hernia 20:43-53. doi:10.1007/s10029-015-1439-5

23. Simons MP, Aufenacker T, Bay-Nielsen M, Bouillot JL, Campanelli G, Conze J, de Lange D, Fortelny R, Heikkinen T, Kingsnorth A, Kukleta J, Morales-Conde S, Nordin P, Schumpelick V, Smedberg S, Smietanski M, Weber G, Miserez M (2009) European Hernia Society guidelines on the treatment of inguinal hernia in adult patients. Hernia 13:343-403. doi:10. 1007/s10029-009-0529-7

24. Bittner R, Arregui ME, Bisgaard T, Dudai M, Ferzli GS, Fitzbibbons RJ, Fortelny RH, Klinge U, Köckerling F, Kuhry E,
Kukleta J, Lomanto D, Misra MC, Montgomery A, MoralesConde S, Reinpold W, Rosenberg J, Sauerland S, Schug-Paß C, Singh K, Timoney M, Weyhe D, Chowbey P (2011) Guidelines for laparoscopic (TAPP) and endoscopic (TEP) treatment of inguinal Hernia [International Endohernia Society (IEHS)]. Surg Endosc 25:2773-2843. doi:10.1007/s00464-011-1799-6

25. Bittner R, Montgomery MA, Arregui E, Bansal V, Bingener J, Bisgaard T, Buhck H, Duai M, Ferzli GS, Fitzgibbons RL, Fortelny RH, Grimes KL, Klinge U, Köckerling F, Kumar S, Kukleta J, Lomanto D, Misra MC, Morales-Conde S, Reinpold W, Rosenberg J, Singh K, Timoney M, Weyhe D, Cowbey P, International Endohernia Society (2015) Update of guidelines on laparoscopic (TAPP) and endoscopic (TEP) treatment of inguinal hernia (International Endohernia Society). Surg Endosc 29:289-321. doi:10.1007/s00464-014-3917-8

26. Poelman MM, van den Heuvel B, Deelder JD, Abis GSA, Beudeker N, Bittner R, Campanelli G, van Dam D, Dwars BJ, Eker $\mathrm{HH}$, Fingerhut A, Khatkov I, Koeckerling F, Kukleta JF, Miserez M, Montgomery A, Munoz Brands RM, Morales-Conde S, Muysoms FE, Soltes M, Tromp W, Yavuz Y, Bonjer HJ (2013) EAES consensus development conference on endoscopic repair of groin hernias. Surg Endosc 27:3505-3519. doi:10.1007/ s00464-013-3001-9

27. Sevonius D, Gunnarsson U, Nordin P, Nilsson E, Sandblom G (2011) Recurrent groin hernia surgery. Br J Surg 98:1489-1494. doi:10.1002/bjs.7559

28. Richards SK, Earnshaw JJ (2003) Management of primary and recurrent inguinal hernia by surgeons from the South West of England. Ann R Coll Surg Engl 85:402-404

29. Stechemesser B, Jacob DA, Schug-Paß C, Köckerling F (2012) Herniamed: an internet-based register for outcome research in hernia surgery. Hernia 16:269-276. doi:10.1007/s10029-0120908-s

30. Miserez M, Alexandre JH, Campanelli G, Corcione F, Cuccurullo D, Pascual MH, Hoeferlin A, Kingsnorth AN, Mandala V, Palot JP, Schumpelick V, Simmermacher RK, Stoppa R, Flament JB (2007) The European hernia society groin hernia classification: simple and easy to remember. Hernia 11:113-116

31. Ollenschläger $G$ (2008) The role of clinical guidelines in medical malpractice litigation. Z. Herz Thorax Gefäßchir. 22:287. doi:10. 1007/s00398-008-0659-8 